\title{
On the theory of the proximity effect in atomic scale superconducting/normal metal multilayered structures
}

\author{
X Montiel ${ }^{1}$ and A I Buzdin ${ }^{2,3}$ \\ ${ }^{1}$ International Institute of Physics, Universidade Federal do Rio Grande do Norte, 59078-400 Natal-RN, \\ Brazil \\ ${ }^{2}$ Condensed Matter Theory Group, LOMA, UMR 5798, Université de Bordeaux I, F-33405 Talence, \\ France \\ ${ }^{3}$ Institut Universitaire de France, France \\ E-mail: x.montiel1@gmail.com and a.bouzdine@loma.u-bordeaux1.fr
}

\begin{abstract}
We determine the influence of the electronic band structure and interlayer coupling on the superconducting properties of quasi-two-dimensional superconductor $(\mathrm{S})$-normal metal $(\mathrm{N})$ nanostructures. An exact analytical solution of the Gor'kov equations which describes the superconducting properties and the local density of states in $\mathrm{S} / \mathrm{N} / \ldots$. $/ \mathrm{N}$ multilayers is found. We study the influence of the electronic spectra, the interlayer coupling and the total number of layers on the local density of states and the superconducting properties of $\mathrm{S} / \mathrm{N} / \ldots / \mathrm{N}$ structures. Moreover, we investigate the impact of the parameters mentioned above on the critical Josephson current in $\mathrm{S} / \mathrm{N} / \ldots$. . N/S junctions.
\end{abstract}

\section{Introduction}

The high-temperature superconductors discovered in 1986 [1] are a good example of natural multilayered superconducting systems. They are characterized by a layered structure [2], strong anisotropic electronic properties [7, 2-6] and can be represented by a stack of alternating superconducting $(\mathrm{S})$, metallic $(\mathrm{N})$ and ferromagnetic $(\mathrm{F})$ layers [2]. These quasi-bidimensional superconducting structures exhibit a strong anisotropy of the magnetic critical field $H_{\mathrm{c} 2}[8,7$, 9-12]. Strongly anisotropic quasi-2D superconductivity was also observed in crystalline organic materials [13, 14]. The most common formalism used to explore the properties of these compounds is the Ginzburg-Landau theory (see for example the [15-18]). Superconducting correlations can be transmitted to non-superconducting materials in contact. This phenomenon, called the proximity effect, influences the superconducting properties of multilayered compounds.

We study the proximity effect in multilayer systems with tunneling coupling composed of an arbitrary number of layers (see figure 1) considered in the framework of the Gor'kov formalism. Discovered in superconducting S/N structures by Meissner [19] and Smith [20], the proximity effect is characterized by the transmission of superconducting correlations into non-superconducting materials. In $\mathrm{N}$ layers, the superconducting correlations decrease exponentially with the distance from the $\mathrm{S} / \mathrm{N}$ interface over the characteristic length $\xi_{n}$, also called the correlation length in the $\mathrm{N}$ layer [21]. The usual values of $\xi_{n}$ are 100-1000 $\AA$, depending on the $\mathrm{N}$ material and temperature. Microscopically, the non-elastic process of Andreev reflection occurs at the $\mathrm{S} / \mathrm{N}$ interface [22, 23]. The proximity effect also develops in $\mathrm{S} / \mathrm{F}$ heterostructures, where the superconducting correlations in the $\mathrm{F}$ layer have an oscillating exponentially decreasing behavior over a characteristic length $\xi_{f}$, which is of order of 10-100 $\AA$ [24-27, 15, 28, 29]. The proximity effect can lead to transmission of Cooper pairs in superconducting junctions, known as the Josephson effect [30, 31].

The proximity effect can strongly influence the properties of the multilayered structure. For example, the competition 


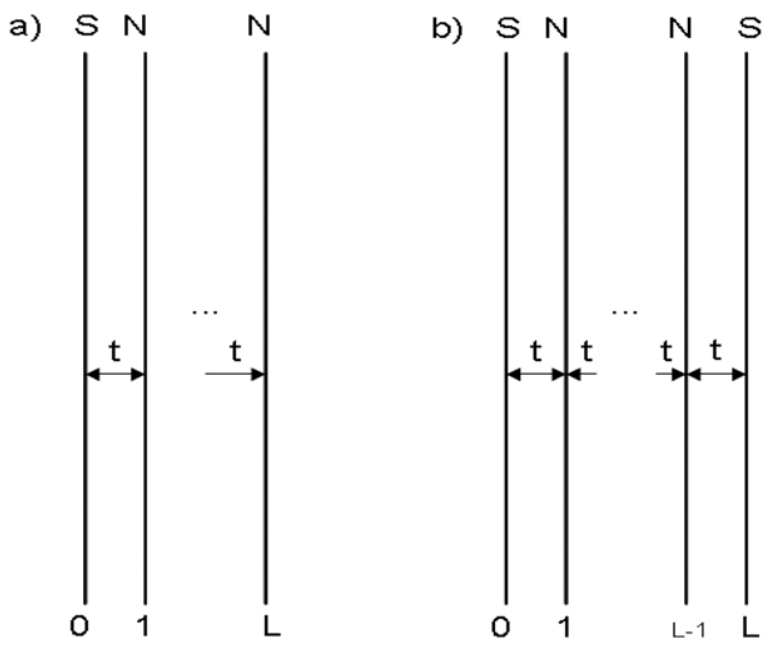

Figure 1. (a) $\mathrm{S} / \mathrm{N} / \ldots / \mathrm{N}$ multilayer of $L$ layers with an interlayer coupling $t$. (b) $\mathrm{S} / \mathrm{N} / \ldots / \mathrm{N} / \mathrm{S}$ Josephson junction of $L$ layers with an interlayer coupling $t$.

between the proximity effect and the Zeeman effect may lead to overcoming of the classical paramagnetic limit for the layered compounds with an elementary unit cell being a $\mathrm{S} / \mathrm{N}$ or $\mathrm{S} / \mathrm{S}$ bilayer, as demonstrated in $[32,33]$. Other unexpected phenomena have been observed in cuprate superconductors.

The proximity effect between the high $T_{\mathrm{c}}$ cuprate superconductors and $\mathrm{N}$ materials exhibits a decay over characteristic lengths much greater than $1000 \AA$. For example, Yuasa et al have observed a proximity effect over a distance about $3000 \AA$ in $\mathrm{Bi}_{2} \mathrm{Sr}_{2} \mathrm{CuO}_{6} / \mathrm{Bi}_{2} \mathrm{Sr}_{2} \mathrm{CaCu}_{2} \mathrm{O}_{8}$ systems [34]. This giant proximity effect has also been observed in other cuprate superconductors/normal metal structures [35-40]. Calculations of the critical Josephson current in $\mathrm{S} / \mathrm{N}^{\prime} / \mathrm{S}$, where $\mathrm{S}$ is a cuprate superconductor and $\mathrm{N}^{\prime}$ a superconductor above its critical temperature, indicate the possible existence of superconducting puddles in the $\mathrm{N}^{\prime}$ layers or superconducting filaments connecting the two $S$ electrodes [41, 42]. Nevertheless, the giant proximity effect exists also in epitaxial $\mathrm{S} / \mathrm{N}$ heterostructures in which the existence of superconducting puddles and inhomogeneities is not possible [43]. The giant proximity effect may result from the intrinsic properties of the $\mathrm{S}$ and the $\mathrm{N}$ layers or the $\mathrm{S} / \mathrm{N}$ structures. Theories based on a triplet superconducting coupling also exist to explain the giant proximity effect in $\mathrm{S} / \mathrm{F}$ structures [44].

Moreover, the density of states (DOS) in $(\mathrm{S} / \mathrm{N})_{n}$ multilayers was shown to have a complex dependence on interlayer coupling [45] and temperature [46]. The DOS and the critical temperature, $T_{\mathrm{c}}$, in the $\mathrm{S} / \mathrm{N}$ bilayer depends on the thickness of the $\mathrm{N}$ layer $[47,48]$. However, the DOS in the $\mathrm{N}$ layer exhibits sub-gap features that cannot be explained by the quasi-classical Usadel equation [49]. The proximity effect may depend on the characteristics of the electronic spectrum [50] and the number of metallic layers in proximity to the $\mathrm{S}$ layer.

Many model used to explain these properties are based on the Bogoliubov-de Gennes theory [21] or on the Eilenberger [51] or on the Usadel formalism [52].
However, these theories cannot describe, in a simple way, the tunneling aspect of the multilayered structure and the details of the electronic spectra in the different layers. We have proposed a way to resolve the system of Gorkov equations of a $\mathrm{S} / \mathrm{N} / \ldots / \mathrm{N}$ multilayer and of a $\mathrm{S} / \mathrm{N} / \ldots / \mathrm{N} / \mathrm{S}$ Josephson junction composed of an arbitrary number of layers $L$ (see figure 1). In this formalism, it is possible to take into account superconducting properties and intrinsic electronic spectra particularities in a layered material. Taking into account these properties could be useful for understanding the physical properties in such multilayers, such as the pseudo-gap and giant proximity effect. In section 1 , we propose a way to resolve the Gorkov equation system for $\mathrm{S} / \mathrm{N} / \ldots / \mathrm{N}$ multilayers (see figure 1(a)) and $\mathrm{S} / \mathrm{N} / \ldots / \mathrm{N} / \mathrm{S}$ Josephson junctions (see figure 1(b)). In section 2, we determine the superconducting properties and the DOS in S/N/N trilayers. Moreover, we study the influence of a mismatch between the $\mathrm{S}$ and $\mathrm{N}$ layer Fermi surface on the DOS in S/N bilayers. Then, we generalize the DOS study to $\mathrm{S} / \mathrm{N} / \ldots / \mathrm{N}$ multilayers in the case of weak interlayer coupling and an energy shifted band structure. In section 5, we study the superconducting gap and the critical Josephson current in weakly coupled S/N/ . . /N/S. We emphasize the crucial role of electronic band structure on the properties of the Josephson junction.

\section{Theory of multilayer system}

We start with a model of alternating superconducting and atomic metallic layers [53-55]. The electrons' motion is described in the $\mathrm{N}$ and $\mathrm{S}$ layers by the spin-independent energy spectrum $\xi_{\mathrm{s}}(\mathbf{k})$ in the $\mathrm{S}$ layers and $\xi_{n}(\mathbf{k})$ in the $\mathrm{N}$ layers. Two basic parameters characterize the system: $t$ is the transfer energy between the $\mathrm{N}$ and $\mathrm{S}$ layers, $\lambda$ is the Cooper pairing constant, which is assumed to be nonzero in $\mathrm{S}$ layers only. It is assumed that the coupling between the layers is realized via the transfer energy $t$, which is relatively small $\left(t \ll E_{\mathrm{F}}\right)$, implying that Cooper pairs are mostly confined inside each layer. In the approximation of linearized electronic spectra, the interlayer coupling can be considered as independent of momentum, as demonstrated in [56]. The Hamiltonian of the system can be written as:

$$
\begin{aligned}
& H=H_{0}+H_{\mathrm{BCS}}+H_{t} \text {, } \\
& H_{0}=\sum_{n, \sigma, \mathbf{k}}\left[\xi_{\sigma, n}(\mathbf{k}) \psi_{\sigma, n}^{+}(\mathbf{k}) \psi_{\sigma, n}(\mathbf{k})\right] \text {, } \\
& H_{\mathrm{BCS}}= \\
& \sum_{n, \mathbf{k}}\left[\Delta_{n}^{*} \psi_{\downarrow, n}^{+}(\mathbf{k}) \psi_{\uparrow, n}^{+}(-\mathbf{k})+\Delta_{n} \psi_{\uparrow, n}(\mathbf{k}) \psi_{\downarrow, n}(-\mathbf{k})\right], \\
& H_{t}=t \sum_{n, \sigma, \mathbf{k}}\left[\psi_{\sigma,(n+1)}^{+}(\mathbf{k}) \psi_{\sigma, n}(\mathbf{k})\right. \\
& \left.+\psi_{\sigma, n}^{+}(\mathbf{k}) \psi_{\sigma,(n+1)}(\mathbf{k})+H . c\right],
\end{aligned}
$$

where $\psi_{\sigma, n}^{+}(\mathbf{k})$ is the creation operator of an electron with spin $\sigma$ and momentum $\mathbf{k}$ in the $n$th layer. The BCS pairing in the $\mathrm{S}$ layer is treated in $H_{\mathrm{BCS}}$ within a mean field approximation [57]. The superconducting order parameter $\Delta_{n}$ is nonzero only in the $\mathrm{S}$ layers. Note that the electrons 
spectra in (1) are calculated from the Fermi energy. As usual, we introduce the normal Green function $G_{\sigma, \sigma^{\prime}}^{n, m}=$ $-\left\langle T_{t}\left(\psi_{\sigma, n}(\mathbf{k}) \psi_{\sigma^{\prime}, m}^{+}(\mathbf{k})\right)\right\rangle$ and anomalous Green functions $F_{\sigma, \sigma^{\prime}}^{\dagger n, m}=\left\langle T_{t}\left(\psi_{\sigma, n}^{+}(\mathbf{k}) \psi_{\sigma^{\prime}, m}^{+}(-\mathbf{k})\right)\right\rangle$ [57], which satisfy the system of equations:

$$
\begin{gathered}
\left(\mathrm{i} \omega-\xi_{\sigma, n}(\mathbf{k})\right) G_{\sigma, \sigma^{\prime}}^{n, m}-t G_{\sigma, \sigma^{\prime}}^{n-1, m} \\
\quad-t G_{\sigma, \sigma^{\prime}}^{n+1, m}+\Delta_{n}^{*} F_{-\sigma, \sigma^{\prime}}^{\dagger n, m}=\delta_{n m} \\
\left(\mathrm{i} \omega+\xi_{-\sigma, n}(\mathbf{k})\right) F_{-\sigma, \sigma^{\prime}}^{\dagger n, m}+t F_{-\sigma, \sigma^{\prime}}^{\dagger n-1, m} \\
\quad+t F_{-\sigma, \sigma^{\prime}}^{\dagger n+1, m}+\Delta_{n} G_{\sigma, \sigma^{\prime}}^{n, m}=0,
\end{gathered}
$$

where $\omega=(2 l+1) \pi T_{\mathrm{c}}$ are the fermionic Matsubara frequencies, and $n$ and $m$ the layer's indices. The superconducting order parameter in the $n$th layer satisfies the standard self-consistency equation

$$
\Delta_{n}^{*}=|\lambda| T \sum_{\omega} \sum_{\mathbf{k}} F_{-\sigma, \sigma^{\prime}}^{\dagger n, n} .
$$

Considering an arbitrary number of layers $L$ (see figure 1 ), the system of equations (2) contains $2 L$ equations. We demonstrate that the Green functions taken in the form

$$
\begin{aligned}
G_{\sigma, \sigma^{\prime}}^{n, m} & =\left[A \mathrm{e}^{q n}+B \mathrm{e}^{-q n}\right] \delta_{\sigma \sigma^{\prime}} \\
F_{-\sigma, \sigma^{\prime}}^{\dagger n, m} & =\left[C \mathrm{e}^{\widetilde{q}^{n}}+D \mathrm{e}^{-\widetilde{q} n}\right] \delta_{\sigma \sigma^{\prime}}
\end{aligned}
$$

are the solution of the system (2) with $m=0, L$. This decomposition has been used on the Green function in the Usadel formalism [48]. $A, B, C$ and $D$ are constants that depend on the other parameters of the studied system. $n$ and $m$ are the layer's indices and $q$ and $\widetilde{q}$ are the wavevectors. The absence of interactions which transform the spin implies an invariance in the spin space. For simplicity, we consider only the case where $\sigma=\uparrow$ and $\sigma^{\prime}=\uparrow$. In the paper, the electronic spectrum in the $\mathrm{S}$ layer $\xi_{\mathrm{s}}$ and in the $\mathrm{N}$ layer $\xi_{n}$ are not assumed to be the same. The multilayer system studied is presented in figure 1 . We may easily see that the solution (4) satisfies the equations (2) when:

$$
\begin{aligned}
\cosh (q) & =\frac{\left(\mathrm{i} \omega-\xi_{n}\right)}{2 t} \\
\widetilde{q} & =q^{*} .
\end{aligned}
$$

Normally in experiments with STM we can only measure the local properties of the first and the last layer of the multilayer. The Green functions which describe the superconducting properties and DOS are $G_{\sigma, \sigma^{\prime}}^{0,0}$ and $F_{-\sigma, \sigma^{\prime}}^{\dagger 0,0}$ in the first layer and $G_{\sigma, \sigma^{\prime}}^{L, L}$ and $F_{-\sigma, \sigma^{\prime}}^{\dagger L, L}$ in the last layer. These functions depend on the parameters of the system, $t, \Delta, \xi_{\mathrm{s}}, \xi_{\mathrm{s}}$ and on the total number of layers $L$.

\subsection{Green function in the $\mathrm{S} / \mathrm{N} / \ldots \mathrm{N}$ multilayer}

We study the $\mathrm{S} / \mathrm{N} / \ldots / \mathrm{N}$ system, where the $\mathrm{S}$ layer has the index $n=0$ and the normal metal layers have indices from $n=1$ to $n=L$ (see figure 1 (a)). The exact solution of the system of Gorkov equations is detailed in the appendix. The Green functions in the $L$ metallic layer can be written as

$$
\begin{aligned}
& G_{\uparrow, \uparrow}^{L, L}=\left\{t^{2} a_{L-1} \tilde{a}_{L}-t\left[\left(\mathrm{i} \omega-\xi_{\mathrm{s}}\right) a_{L} \widetilde{a}_{L}-\left(\mathrm{i} \omega+\xi_{\mathrm{s}}\right) a_{L-1} \widetilde{a}_{L+1}\right]\right. \\
& \left.\quad+\left(\omega^{2}+\xi_{\mathrm{s}}^{2}+\left|\Delta_{0}\right|^{2}\right) a_{L} \widetilde{a}_{L+1}\right\}\left\{t \left(t^{2} a_{L} \widetilde{a}_{L}\right.\right. \\
& \quad-t\left[\left(\mathrm{i} \omega-\xi_{\mathrm{s}}\right) a_{L+1} \widetilde{a}_{L}-\left(\mathrm{i} \omega+\xi_{\mathrm{s}}\right) a_{L} \widetilde{a}_{L+1}\right] \\
& \left.\left.\quad+\left(\omega^{2}+\xi_{\mathrm{s}}^{2}+\left|\Delta_{0}\right|^{2}\right) a_{L+1} \widetilde{a}_{L+1}\right)\right\}^{-1} \\
& \quad F_{\downarrow, \uparrow}^{\dagger L, L}=\left(\Delta_{0}^{*} a_{1} \widetilde{a}_{1}\right)\left\{\left(t^{2} a_{L} \widetilde{a}_{L}-t\left[\left(\mathrm{i} \omega-\xi_{\mathrm{s}}\right) a_{L+1} \widetilde{a}_{L}\right.\right.\right. \\
& \left.\quad-\left(\mathrm{i} \omega+\xi_{\mathrm{s}}\right) a_{L} \tilde{a}_{L+1}\right] \\
& \left.\left.\quad+\left(\omega^{2}+\xi_{\mathrm{s}}^{2}+\left|\Delta_{0}\right|^{2}\right) a_{L+1} \widetilde{a}_{L+1}\right)\right\}^{-1}
\end{aligned}
$$

where $a_{n}=\sinh (q n)$ and $\tilde{a}_{n}=\sinh (\tilde{q} n)$. The Green functions in the superconducting layer can be written as

$$
\begin{aligned}
& F_{\downarrow, \uparrow}^{\dagger 0,0}=\left\{\Delta_{0}^{*} a_{L+1} \widetilde{a}_{L+1}\right\}\left\{\left(\omega^{2}+\xi_{\mathrm{s}}^{2}+\left|\Delta_{0}\right|^{2}\right) a_{L+1} \widetilde{a}_{L+1}\right. \\
& \left.\quad-t\left[\left(\mathrm{i} \omega-\xi_{\mathrm{s}}\right) a_{L+1} \widetilde{a}_{L}-\left(\mathrm{i} \omega+\xi_{\mathrm{s}}\right) a_{L} \widetilde{a}_{L+1}\right]+t^{2} a_{L} \widetilde{a}_{L}\right\}^{-1} \\
& G_{\uparrow, \uparrow}^{0,0}=\left\{-a_{L+1}\left[\left(\mathrm{i} \omega+\xi_{\mathrm{s}}\right) \tilde{a}_{L+1}+\tilde{a}_{L}\right]\right\}\left\{\left(\omega^{2}+\xi_{\mathrm{s}}^{2}\right.\right. \\
& \left.\quad+\left|\Delta_{0}\right|^{2}\right) a_{L+1} \widetilde{a}_{L+1}-t\left[\left(\mathrm{i} \omega-\xi_{\mathrm{s}}\right) a_{L+1} \widetilde{a}_{L}\right. \\
& \left.\left.\quad-\left(\mathrm{i} \omega+\xi_{\mathrm{s}}\right) a_{L} \tilde{a}_{L+1}\right]+t^{2} a_{L} \widetilde{a}_{L}\right\}^{-1}
\end{aligned}
$$

From the Green function (9), it is possible to determine the superconducting properties as a function of the total number of layers $L$. The dependence on $L$ of the Green function is nontrivial. However, it is possible to transform the coefficients $a_{L}$ and $\tilde{a}_{L}$ by their explicit forms (see formula (A.2) in the appendix). This explicit form takes into account the exponential form of the coefficients $a_{L}$ and $\widetilde{a}_{L}$, which depend on $\xi_{\mathrm{s}}, \xi_{n}, t$ and $L$. In section 3, we study the case of the bilayer $(L=1)$ and of the trilayer $(L=2)$. The multilayer is studied in the case of weak interlayer coupling $t \ll T_{\mathrm{c} 0}$, where $T_{\mathrm{c} 0}$ is the critical temperature of a single $\mathrm{S}$ layer. This temperature is related to the superconducting gap at zero temperature $\Delta(0)$ by the relation $\Delta(0)=1764 T_{\mathrm{c} 0}$.

\subsection{Gorkov functions in the $\mathrm{S} / \mathrm{N} / \ldots$. N/S Josephson junction}

We study the Josephson critical current in a S/N/S junction with an arbitrary thickness. In this $\mathrm{S} / \mathrm{N} / \mathrm{S}$ system, the superconducting layers have the indices $n=0$ and $n=L$ whereas the metallic layers have all the indices from $n=1$ to $n=L-1$ (see figure 1(b)). The exact solution of the system of Gorkov equations is detailed in the appendix. The anomalous and normal Green functions in the last superconducting layer can be written as

$$
\begin{aligned}
& G_{\uparrow, \uparrow}^{L, L}=\left\{-t^{3} S_{L-1}^{L-2}-t^{2}\left[\widetilde{\omega}^{*} S_{L}^{L-2}+2 \widetilde{\omega} S_{L-1}^{L-1}\right]\right. \\
& \left.\quad-t\left[S_{L}^{L-1}\left(|\widetilde{\omega}|^{2}+\Omega_{0}^{2}\right)+\widetilde{\omega}^{2} S_{L-1}^{L}\right]-\widetilde{\omega} \Omega_{0}^{2} S_{L}^{L}\right\} \\
& \quad \times\left\{t^{4} S_{L-2}^{L-2}+2 t^{3} X_{L-2}^{L-1}+t^{2}\left[\left(2|\widetilde{\omega}|^{2}+\Omega_{0}^{2}+\Omega_{L}^{2}\right) S_{L-1}^{L-1}\right.\right. \\
& \left.\quad+2 \Delta_{0} \Delta_{L} S_{1}^{1}+\widetilde{\omega}^{*^{2}} S_{L-2}^{L}+\widetilde{\omega}^{2} S_{L-2}^{L}\right] \\
& \left.\quad+t\left(\Omega_{0}^{2}+\Omega_{L}^{2}\right) X_{L-1}^{L}+S_{L}^{L} \Omega_{L}^{2} \Omega_{0}^{2}\right\}^{-1} \\
& F_{\downarrow, \uparrow}^{\dagger L, L}=\left\{t^{2} \Delta_{0}^{*} S_{1}^{1}+\Delta_{L}^{*}\left\{\Omega_{0}^{2} S_{L}^{L}+t^{2} S_{L}^{L-1}-t \widetilde{\omega}^{*} S_{L-1}^{L}\right.\right. \\
& \left.\left.\quad-t \widetilde{\omega} S_{L-1}^{L}\right\}\right\}\left\{t^{4} S_{L-2}^{L-2}+2 t^{3} X_{L-2}^{L-1}\right.
\end{aligned}
$$




$$
\begin{aligned}
& +t^{2}\left[\left(2|\widetilde{\omega}|^{2}+\Omega_{0}^{2}+\Omega_{L}^{2}\right)\right. \\
& \left.\times S_{L-1}^{L-1}+2 \Delta_{0} \Delta_{L} S_{1}^{1}+\widetilde{\omega}^{*^{2}} S_{L-2}^{L}+\widetilde{\omega}^{2} S_{L-2}^{L}\right] \\
& \left.+t\left(\Omega_{0}^{2}+\Omega_{L}^{2}\right) X_{L-1}^{L}+S_{L}^{L} \Omega_{L}^{2} \Omega_{0}^{2}\right\}^{-1}
\end{aligned}
$$

where $a_{n}=\sinh (q n)$ and $\widetilde{a}_{n}=\sinh (\widetilde{q} n), S_{\mathrm{N}}^{\mathrm{M}}=\widetilde{a}_{\mathrm{M}} a_{\mathrm{N}}, \widetilde{\omega}=$ $\mathrm{i} \omega+\xi_{\mathrm{s}}, \widetilde{\omega}^{*}=-\mathrm{i} \omega+\xi_{\mathrm{s}}=-\left(\mathrm{i} \omega-\xi_{\mathrm{s}}\right), \Omega_{0}^{2}=|\widetilde{\omega}|^{2}+\Delta_{0}^{2}, \Omega_{L}^{2}=$ $|\widetilde{\omega}|^{2}+\Delta_{L}^{2}$ and $X_{\mathrm{M}}^{N}=\widetilde{\omega} S_{\mathrm{M}}^{N}+\widetilde{\omega}^{*} S_{\mathrm{N}}^{\mathrm{M}}$. In the first superconducting layer, the Green functions can be written as

$$
\begin{aligned}
& G_{\uparrow, \uparrow}^{0,0}=\left\{-t^{3} S_{L-1}^{L-2}-t^{2}\left[\widetilde{\omega}^{*} S_{L}^{L-2}+2 \widetilde{\omega} S_{L-1}^{L-1}\right]\right. \\
&\left.-t\left[S_{L}^{L-1}\left(|\widetilde{\omega}|^{2}+\Omega_{L}^{2}\right)+\widetilde{\omega}^{2} S_{L-1}^{L}\right]-\widetilde{\omega} \Omega_{L}^{2} S_{L}^{L}\right\} \\
& \times\left\{t^{4} S_{L-2}^{L-2}+2 t^{3} X_{L-2}^{L-1}+t^{2}\left[\left(2|\widetilde{\omega}|^{2}+\Omega_{0}^{2}+\Omega_{L}^{2}\right) S_{L-1}^{L-1}\right.\right. \\
&\left.+2 \Delta_{0} \Delta_{L} S_{1}^{1}+\widetilde{\omega}^{*^{2}} S_{L-2}^{L}+\widetilde{\omega}^{2} S_{L-2}^{L}\right] \\
&\left.+t\left(\Omega_{0}^{2}+\Omega_{L}^{2}\right) X_{L-1}^{L}+S_{L}^{L} \Omega_{L}^{2} \Omega_{0}^{2}\right\}^{-1} \\
& F_{\downarrow, \uparrow}^{\dagger 0,0}=\left\{t^{2} \Delta_{L}^{*} S_{1}^{1}+\Delta_{0}^{*}\left\{\Omega_{L}^{2} S_{L}^{L}+t^{2} S_{L}^{L-1}-t \widetilde{\omega}^{*} S_{L-1}^{L}\right.\right. \\
&\left.\left.\quad-t \widetilde{\omega} S_{L-1}^{L}\right\}\right\}\left\{t^{4} S_{L-2}^{L-2}+2 t^{3} X_{L-2}^{L-1}\right. \\
& \quad+t^{2}\left[\left(2|\widetilde{\omega}|^{2}+\Omega_{0}^{2}+\Omega_{L}^{2}\right) S_{L-1}^{L-1}\right. \\
&\left.\quad+2 \Delta_{0} \Delta_{L} S_{1}^{1}+\widetilde{\omega}^{*^{2}} S_{L-2}^{L}+\widetilde{\omega}^{2} S_{L-2}^{L}\right] \\
&\left.\quad+t\left(\Omega_{0}^{2}+\Omega_{L}^{2}\right) X_{L-1}^{L}+S_{L}^{L} \Omega_{L}^{2} \Omega_{0}^{2}\right\}^{-1} .
\end{aligned}
$$

The dependence on $L$ of the Green function is complex. From the relations (6) and (5) it is possible to determine the explicit form of the Green functions. In section 4, we analyze the Josephson physics in the case of an arbitrary number of layers $L$ in the case of weak interlayer coupling $t \ll T_{\mathrm{c} 0}$.

\section{The proximity effect in $\mathrm{S} / \mathrm{N} / \ldots / \mathrm{N}$ multilayers}

From the Green functions in the $\mathrm{S}$ layer $(n=0)$ and in the last metal layer $(n=L)$ of an $\mathrm{S} / \mathrm{N} / \ldots / \mathrm{N}$ multilayer, we determine the density of states in the $\mathrm{S}$ layer and in the last metal layer in several cases. First, we find the density of states and superconducting properties of the $\mathrm{S} / \mathrm{N} / \mathrm{N}$ trilayer. Then, we study the influence of a mismatch of $\mathrm{S}$ and $\mathrm{N}$ layer Fermi surfaces on the properties of the S/N bilayer. To finish, we generalize these results to the properties of the $\mathrm{S} / \mathrm{N} / \ldots / \mathrm{N}$ multilayer in the case of weak interlayer coupling $t \ll T_{\mathrm{c} 0}$. The local density of states in the layer $n$ is determined from the relation

$$
\rho^{n}(E)=-2 \operatorname{Im} \int_{-\infty}^{+\infty} \lim _{\delta \rightarrow 0} G_{\uparrow, \uparrow}^{n, n}(E+\mathrm{i} \delta, \xi) \mathrm{d} \xi .
$$

\subsection{The $\mathrm{S} / \mathrm{N} / \mathrm{N}$ trilayer}

We will study the thermodynamic properties and the density of states in the S/N/N trilayer. From the Hamiltonian (1) and the definition of the Green functions we deduce the Gorkov system of the $\mathrm{S} / \mathrm{N} / \mathrm{N}$ trilayer as

$$
\begin{gathered}
\left(\mathrm{i} \omega-\xi_{n}\right) G_{\uparrow, \uparrow}^{2,2}-t G_{\uparrow, \uparrow}^{1,2}=1 \\
\left(\mathrm{i} \omega-\xi_{n}\right) G_{\uparrow, \uparrow}^{1,2}-t G_{\uparrow, \uparrow}^{2,2}-t G_{\uparrow, \uparrow}^{0,2}=0 \\
\left(\mathrm{i} \omega-\xi_{\mathrm{s}}\right) G_{\uparrow, \uparrow}^{0,2}-t G_{\uparrow, \uparrow}^{1,2}+\Delta_{0} F_{\downarrow, \uparrow}^{\dagger 0,2}=0 \\
\left(\mathrm{i} \omega+\xi_{\mathrm{s}}\right) F_{\downarrow, \uparrow}^{\dagger 0,2}+t F_{\downarrow, \uparrow}^{\dagger 1,2}+\Delta_{0} G_{\uparrow, \uparrow}^{0,2}=0 \\
\left(\mathrm{i} \omega+\xi_{n}\right) F_{\downarrow, \uparrow}^{\dagger 1,2}+t F_{\downarrow, \uparrow}^{\dagger 0,2}+t F_{\downarrow, \uparrow}^{\dagger 2,2}=0 \\
\left(\mathrm{i} \omega+\xi_{n}\right) F_{\downarrow, \uparrow}^{\dagger 2,2}+t F_{\downarrow, \uparrow}^{\dagger 1,2}=0
\end{gathered}
$$

where we can determine the normal and anomalous Green functions of the metal layer with the index $n=2$. The normal Green function in the $\mathrm{N}$ layer $(n=2)$ is written as:

$$
\begin{aligned}
G_{\uparrow, \uparrow}^{2,2}= & \left\{\left((\mathrm{i} \omega+\xi)^{2}-t^{2}\right)(\mathrm{i} \omega-\xi) \Delta^{2}-\left((\mathrm{i} \omega-\xi)^{2}-t^{2}\right)\right. \\
& \left.\times\left((\mathrm{i} \omega+\xi)^{2}-2 t^{2}\right)(\mathrm{i} \omega+\xi)\right\}\left\{\left((\mathrm{i} \omega+\xi)^{2}-t^{2}\right)\right. \\
& \times\left((\mathrm{i} \omega-\xi)^{2}-t^{2}\right) \Delta^{2}+\left(\omega^{2}+\xi^{2}\right) \\
& \left.\times\left((\mathrm{i} \omega+\xi)^{2}-2 t^{2}\right)\left((\mathrm{i} \omega-\xi)^{2}-2 t^{2}\right)\right\}^{-1} .
\end{aligned}
$$

The normal Green function indexed $n=1$ is written as

$$
\begin{aligned}
G_{\uparrow, \uparrow}^{1,1}= & \left\{\left(\left(\omega^{2}+\xi^{2}\right)\left((\mathrm{i} \omega+\xi)^{2}-2 t^{2}\right)+\Delta^{2}\left((\mathrm{i} \omega+\xi)^{2}\right.\right.\right. \\
& \left.\left.\left.-t^{2}\right)\right)(\mathrm{i} \omega-\xi)\right\}\left\{( ( \mathrm { i } \omega + \xi ) ^ { 2 } - t ^ { 2 } ) \left((\mathrm{i} \omega-\xi)^{2}\right.\right. \\
& \left.-t^{2}\right) \Delta^{2}+\left(\omega^{2}+\xi^{2}\right)\left((\mathrm{i} \omega+\xi)^{2}-2 t^{2}\right) \\
& \left.\times\left((\mathrm{i} \omega-\xi)^{2}-2 t^{2}\right)\right\}^{-1}
\end{aligned}
$$

and the Green function in the superconducting layer indexed $n=0$ is

$$
\begin{aligned}
& G_{\uparrow, \uparrow}^{0,0}=\left\{-\left((\mathrm{i} \omega-\xi)^{2}-t^{2}\right)\left((\mathrm{i} \omega+\xi)^{2}-2 t^{2}\right)(\mathrm{i} \omega+\xi)\right\} \\
& \quad \times\left\{\left((\mathrm{i} \omega+\xi)^{2}-t^{2}\right)\left((\mathrm{i} \omega-\xi)^{2}-t^{2}\right) \Delta^{2}+\left(\omega^{2}+\xi^{2}\right)\right. \\
& \left.\quad \times\left((\mathrm{i} \omega+\xi)^{2}-2 t^{2}\right)\left((\mathrm{i} \omega-\xi)^{2}-2 t^{2}\right)\right\}^{-1} \\
& F_{\downarrow, \uparrow}^{\dagger 0,0}=\left\{-\left(\left(\mathrm{i} \omega+\xi_{\mathrm{s}}\right)^{2}-t^{2}\right)\left(\left(\mathrm{i} \omega-\xi_{\mathrm{s}}\right)^{2}-t^{2}\right) \Delta^{*}\right\} \\
& \quad \times\left\{\left((\mathrm{i} \omega+\xi)^{2}-t^{2}\right)\left((\mathrm{i} \omega-\xi)^{2}-t^{2}\right) \Delta^{2}+\left(\omega^{2}+\xi^{2}\right)\right. \\
& \left.\quad \times\left((\mathrm{i} \omega+\xi)^{2}-2 t^{2}\right)\left((\mathrm{i} \omega-\xi)^{2}-2 t^{2}\right)\right\}^{-1}
\end{aligned}
$$

Using these functions, we can calculate the critical temperature, the superconducting gap and the density of states in all the layers of the $\mathrm{S} / \mathrm{N} / \mathrm{N}$ trilayer. We note that we can obtain the Green functions (17) and (19) by putting $L=2$ in the general expressions (7) and (10) respectively.

3.1.1. Critical temperature of the $S$ layer as a function of $t$. From the self-consistency equation (3), using the anomalous Green function (20), we obtain that the critical superconducting temperature is the solution of the equation:

$$
\begin{aligned}
\ln \left(\frac{T_{\mathrm{c}}}{T_{\mathrm{c} 0}}\right)= & -\frac{1}{4}\left[\frac{5}{2} \gamma+5 \ln (2)+\frac{1}{4} \Psi\left(\frac{1}{2}-\mathrm{i} \frac{\sqrt{2} t}{\pi T_{\mathrm{c}}}\right)\right. \\
& +\frac{1}{4} \Psi\left(\frac{1}{2}+\mathrm{i} \frac{\sqrt{2} t}{\pi T_{\mathrm{c}}}\right)+\Psi\left(\frac{1}{2}-\mathrm{i} \frac{\sqrt{2} t}{4 \pi T_{\mathrm{c}}}\right) \\
& \left.+\Psi\left(\frac{1}{2}+\mathrm{i} \frac{\sqrt{2} t}{4 \pi T_{\mathrm{c}}}\right)\right] .
\end{aligned}
$$

The dependence of the critical temperature on the interlayer coupling is represented in figure 2 by the solid line. 


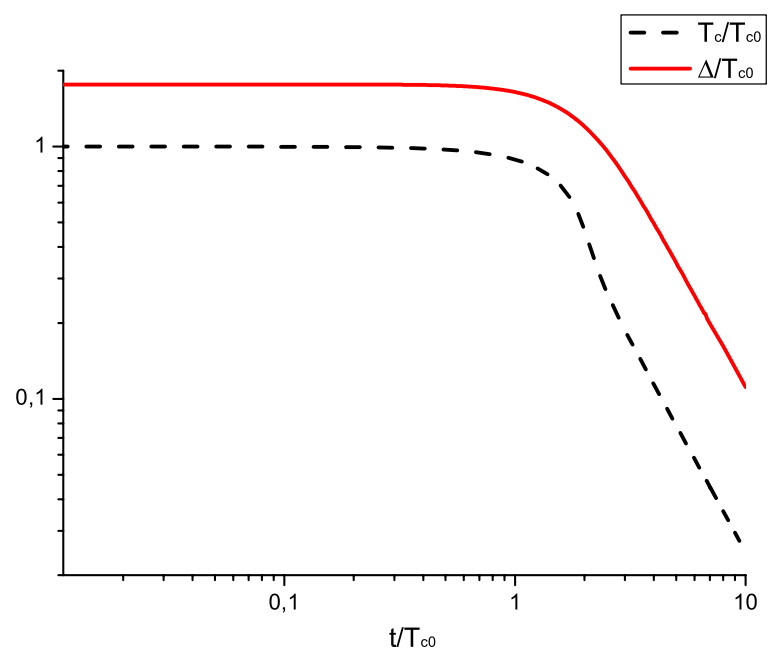

Figure 2. Graph of $T_{\mathrm{c}} / T_{\mathrm{c} 0}$ and $\Delta / T_{\mathrm{c} 0}$ as a function of $t / T_{\mathrm{c} 0}$ (solid line). For $t \gg T_{\mathrm{c} 0}$, the critical temperature and the superconducting gap at zero temperature of the $\mathrm{S}$ layer decrease with the interlayer coupling.

In the weak interlayer coupling regime, $t \ll T_{\mathrm{c} 0}$, the superconducting critical temperature varies as $\frac{T_{\mathrm{c}}}{T_{\mathrm{c} 0}}=1-$ $\frac{7}{8} \frac{\zeta(3)}{\pi^{2}}\left(\frac{t}{T_{c 0}}\right)^{2}$, which is the same decay as observed in the $\mathrm{S} / \mathrm{N}$ bilayer [32]. In the strong interlayer coupling regime, $t \gg$ $T_{\mathrm{c} 0}$, the superconducting critical temperature varies as $\frac{T_{\mathrm{c}}}{T_{\mathrm{c} 0}}=$ $\left(\frac{\mathrm{e}^{-\gamma} \pi T_{\mathrm{c} 0}}{4 \sqrt{2} t}\right)^{\frac{5}{3}}$. The power law decay is larger than in the $\mathrm{S} / \mathrm{N}$ bilayer [32]. In the trilayer, the $S$ layer transmits its correlation in two normal metal layers. The superconducting state is more destabilized in the trilayer than in the bilayer [32].

\subsubsection{Superconducting gap at zero temperature.} superconducting gap at zero temperature can be calculated with the help of the anomalous Green function of the $\mathrm{S}$ layer (20). The self-consistency equation (3) is written as:

$$
\begin{aligned}
\ln \left(\frac{\Delta(0)}{\Delta_{0}}\right)= & \frac{1}{2 \pi} \int_{0}^{\infty} \mathrm{d} \rho \int_{0}^{2 \pi} \mathrm{d} \phi\left(\left(t^{4}+2 \rho\left(\sin ^{2}(\phi)\right.\right.\right. \\
& \left.\left.-\cos ^{2}(\phi)\right) t^{2}+\rho^{2}\right)\left(\left(4 \rho+\Delta^{2}\right) t^{4}\right. \\
& +2 \rho\left(\sin ^{2}(\phi)-\cos ^{2}(\phi)\right)\left(2 \rho+\Delta^{2}\right) t^{2} \\
& \left.\left.+\rho^{2}\left(\rho+\Delta^{2}\right)\right)^{-1}-\frac{1}{\rho+|\Delta|^{2}}\right)
\end{aligned}
$$

where $\Delta(0)$ is the superconducting gap of the $\mathrm{S}$ layer at zero temperature and with the variable change $\xi_{\mathrm{s}}=\sqrt{\rho} \sin (\phi)$ and $\omega=\sqrt{\rho} \cos (\phi)$. The results are presented in figure 2 . The variation of the superconducting gap at zero temperature is globally the same as for the critical temperature. But the proportionality coefficient between $\Delta(0)$ and $T_{\mathrm{c}}$ evolves with the interlayer coupling from $\left(\Delta(0) / T_{\mathrm{c}}\right)=1.764$ at zero interlayer coupling to $\left(\Delta(0) / T_{\mathrm{c}}\right)=4.51$ at high interlayer coupling (see figure 3 ). This result is coherent with [45], where it has been demonstrated that the ratio $\left(\Delta(0) / T_{\mathrm{c}}\right)$ in the $\mathrm{S} / \mathrm{N}$ bilayer evolves from 1.764 at small interlayer coupling to 3.52 at high interlayer coupling.

Then the ratio $\left(\Delta(0) / T_{\mathrm{c}}\right)$ depends on the number of layers composing the multilayer at high interlayer coupling.

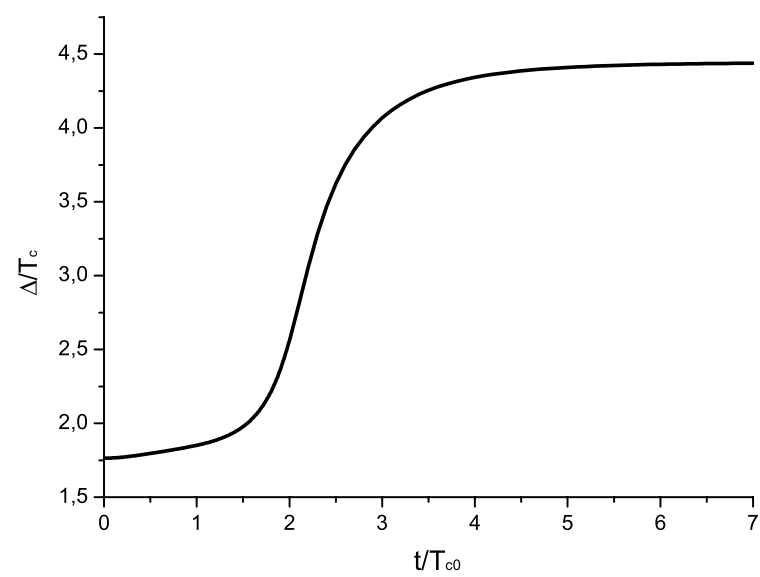

Figure 3. Graph of $\Delta(0) / T_{\mathrm{c}}$ as a function of $t / T_{\mathrm{c} 0}$. The value of $\Delta(0) / T_{\mathrm{c}}$ evolves from 1.764 for weak interlayer coupling $\left(t \ll T_{\mathrm{c} 0}\right)$ to 4.51 for strong interlayer coupling $\left(t \gg T_{\mathrm{c} 0}\right)$.

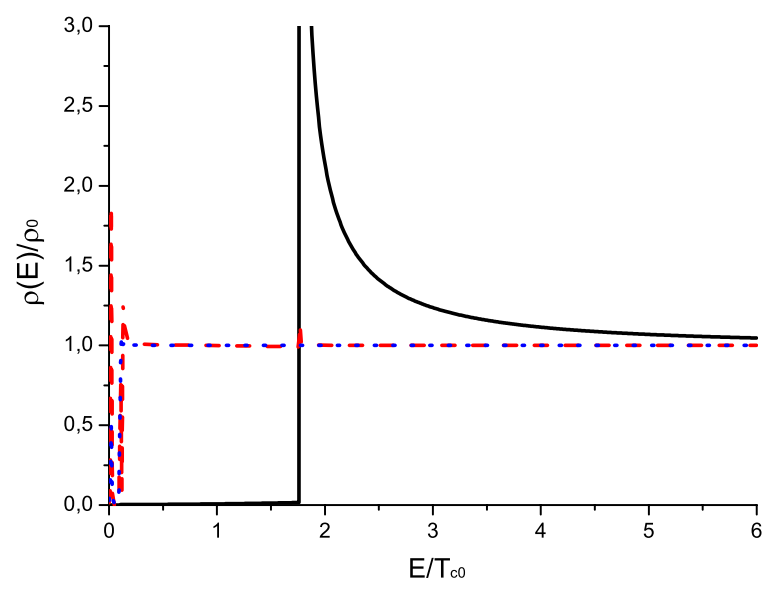

Figure 4. Density of states $\rho(E) / \rho(0)$ in the superconducting layer (solid line), in the first normal metal layer (dashed line) and in the second normal metal layer (dotted line) in the case $t=0.1 T_{\mathrm{c} 0}$.

It seems to increase with the total number of layers in the $\mathrm{S} / \mathrm{N}$ structure. The superconductivity is strongly weakened in the presence of a large number of $\mathrm{N}$ layers.

3.1.3. Density of states in the S/N/N trilayer. The DOS inside each layer of the $\mathrm{S} / \mathrm{N} / \mathrm{N}$ trilayer is deduced from the normal Green function inside the layers, (17)-(19). We determine the DOS from the relation (15) in the superconducting layer and in the two normal metal layers for different interlayer couplings. The different DOS are determined numerically and presented in figures $4-8$. In the different graphs, we note the presence of different peaks in the DOS. As in [45], these peaks arise at energies which depend on the superconducting gap $\Delta$ and the interlayer coupling $t$. In the trilayers, we emphasize the presence of three peaks because of the presence of three layers in the system. In the weak coupling approximation, $t \ll \Delta$, two peaks arise at energy $E_{\mathrm{a}} \approx t$. These peaks are related to the hybridization levels that occur with interlayer coupling. The third peak appears at an energy around $E_{\mathrm{b}} \approx \Delta$ which is related to the superconducting state in the $S$ layer. In the strong coupling approximation, $t \gg \Delta$, the two peaks 


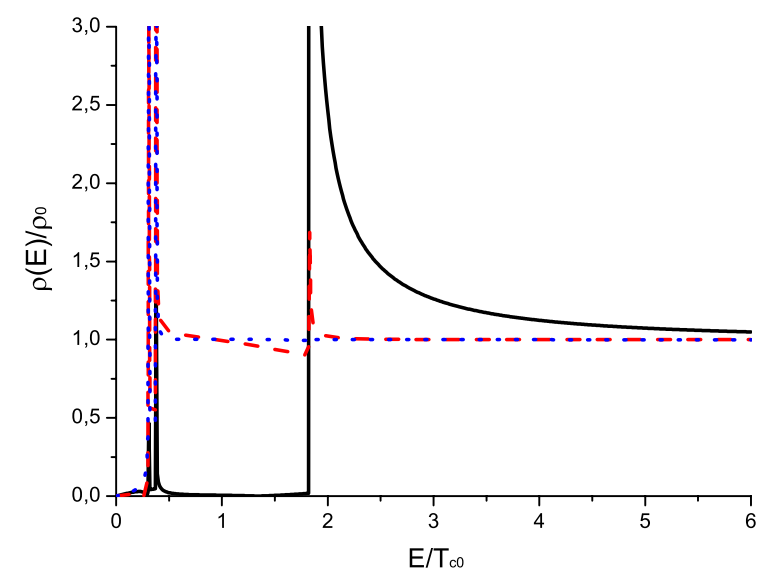

Figure 5. Density of states $\rho(E) / \rho(0)$ in the superconducting layer (solid line), in the first normal metal layer (dashed line) and in the second normal metal layer (dotted line) in the case $t=0.35 T_{\mathrm{c} 0}$.

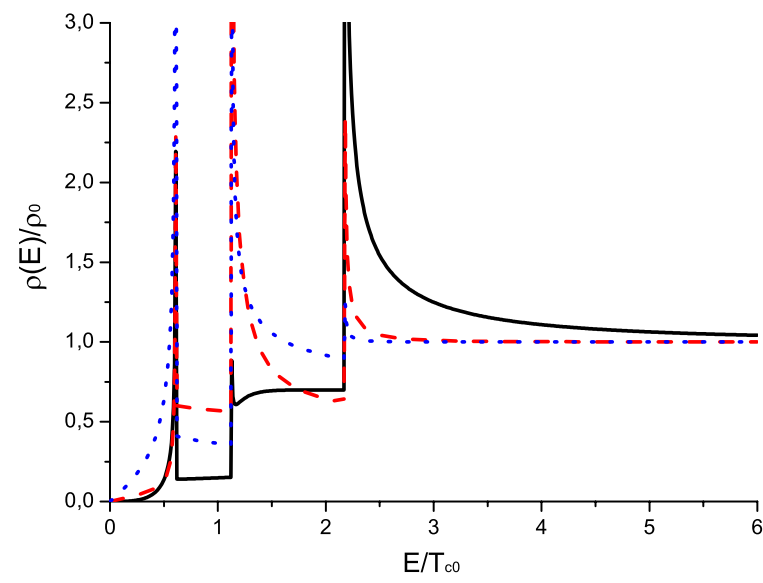

Figure 6. Density of states $\rho(E) / \rho(0)$ in the superconducting layer (solid line), in the first normal metal layer (dashed line) and in the second normal metal layer (dotted line) in the case $t=0.95 T_{\mathrm{c} 0}$.

due to the hybridization levels arise at energy $E_{\mathrm{a}} \approx \sqrt{2} t$. The third peak related to the superconducting state in the $\mathrm{S}$ layer appears at energy $E_{\mathrm{b}} \approx \frac{\Delta}{2}$. At high energies, the DOS in the $\mathrm{S} / \mathrm{N} / \mathrm{N}$ layers equals the DOS in the metal layer.

\subsection{Dependence of a mismatch between the $N$ and $S$ layer Fermi surface on the properties of a $S / N$ bilayer}

The dependence of a mismatch between the $\mathrm{N}$ and $\mathrm{S}$ layer Fermi surface on the properties of a $\mathrm{S} / \mathrm{F} / \mathrm{S}$ junction and a $\mathrm{F} / \mathrm{S} / \mathrm{F}$ trilayer has been studied in [50]. In this paper, we demonstrate that an energy shift between the superconductor and the normal metal qualitatively affect the properties of the heterostructure. In this case, we study the influence of an energy shift on the properties of a S/N bilayer. The electronic spectrum near the Fermi surface in the superconducting layer is $\xi_{\mathrm{s}}$, whereas the metallic spectrum is $\xi_{n}=\xi_{\mathrm{s}}+\Delta E$, where $\Delta E$ is the energy shift due to the mismatch of the Fermi surface of $\mathrm{N}$ and $\mathrm{S}$ layers. The normal and anomalous Green functions in the $\mathrm{S}$ layer are deduced from the formulas (9) and

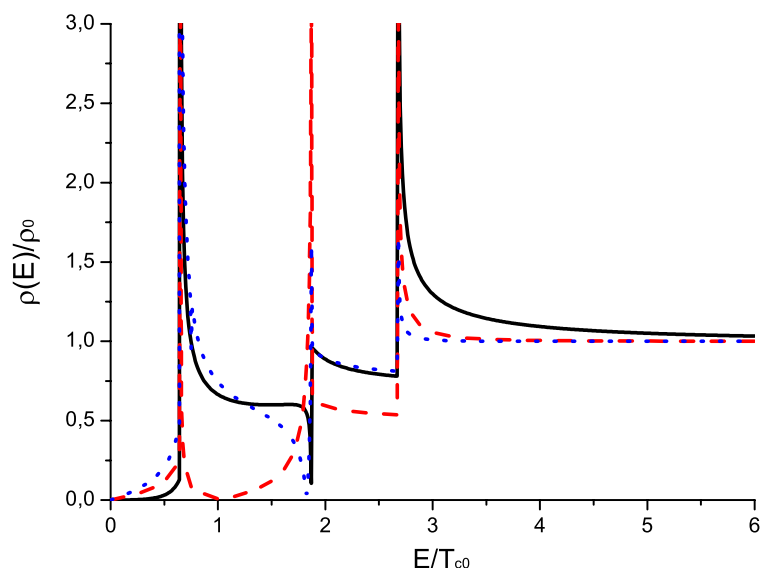

Figure 7. Density of states $\rho(E) / \rho(0)$ in the superconducting layer (solid line), in the first normal metal layer (dashed line) and in the second normal metal layer (dotted line) in the case $t=1.5 T_{\mathrm{c} 0}$.

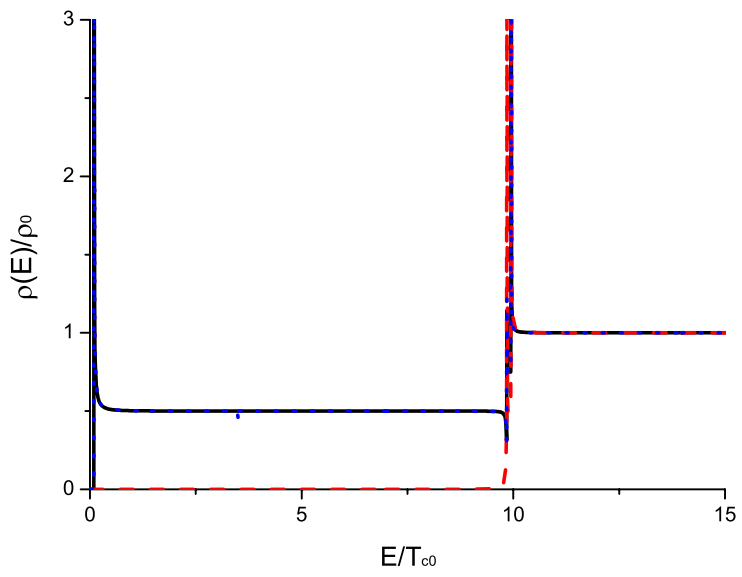

Figure 8. Density of states $\rho(E) / \rho(0)$ in the superconducting layer (solid line), in the first normal metal layer (dashed line) and in the second normal metal layer (dotted line) in the case $t=7 T_{\mathrm{c} 0}$.

(10) by setting $L=1$ :

$$
\begin{aligned}
& G_{\uparrow, \uparrow}^{0,0}=\left\{\left(\mathrm{i} \omega-\xi_{\mathrm{s}}-\Delta E\right)\left(\left(\mathrm{i} \omega+\xi_{\mathrm{s}}+\Delta E\right)\left(\mathrm{i} \omega-\xi_{\mathrm{s}}\right)-t^{2}\right)\right\} \\
& \quad \times\left\{t^{4}+\left(2 \omega^{2}-2 \xi_{\mathrm{s}}\left(\xi_{\mathrm{s}}+\Delta E\right)\right) t^{2}+\left(\omega^{2}+\left(\xi_{\mathrm{s}}+\Delta E\right)^{2}\right)\right. \\
& \left.\quad \times\left(\omega^{2}+\xi_{\mathrm{s}}^{2}+\Delta^{2}\right)\right\}^{-1} \\
& F_{\downarrow, \uparrow}^{+0,0}=-\left\{\Delta^{*}\left(\omega^{2}+\left(\xi_{\mathrm{s}}+\Delta E\right)^{2}\right)\right\}\left\{t^{4}+\left(2 \omega^{2}-2 \xi_{\mathrm{s}}\left(\xi_{\mathrm{s}}\right.\right.\right. \\
& \left.\quad+\Delta E)) t^{2}+\left(\omega^{2}+\left(\xi_{\mathrm{s}}+\Delta E\right)^{2}\right)\left(\omega^{2}+\xi_{\mathrm{s}}^{2}+\Delta^{2}\right)\right\}^{-1} .
\end{aligned}
$$

The normal Green function in the metallic layer is deduced from the function (7) by replacing $L=1$ :

$$
\begin{aligned}
G_{\uparrow, \uparrow}^{1,1}= & \left\{-\left(\mathrm{i} \omega-\xi_{\mathrm{s}}-\Delta E\right)\left(\mathrm{i} \omega-\xi_{\mathrm{s}}\right) t^{2}+\left(\omega^{2}+\left(\xi_{\mathrm{s}}+\Delta E\right)^{2}\right)\right. \\
& \left.\times\left(\omega^{2}+\xi_{\mathrm{s}}^{2}+\Delta^{2}\right)\right\}\left\{\left(\mathrm{i} \omega-\xi_{\mathrm{s}}-\Delta E\right)\right. \\
& \times\left(t^{4}+\left(2 \omega^{2}-2 \xi_{\mathrm{s}}\left(\xi_{\mathrm{s}}+\Delta E\right) t^{2}\right.\right. \\
& \left.\left.\left.+\left(\omega^{2}+\left(\xi_{\mathrm{s}}+\Delta E\right)^{2}\right)\left(\omega^{2}+\xi_{\mathrm{s}}^{2}+\Delta^{2}\right)\right)\right)\right\}^{-1}
\end{aligned}
$$

We study the critical temperature and the density of states of the $\mathrm{S}$ and the $\mathrm{N}$ layers for an arbitrary energy shift $\Delta E$. 


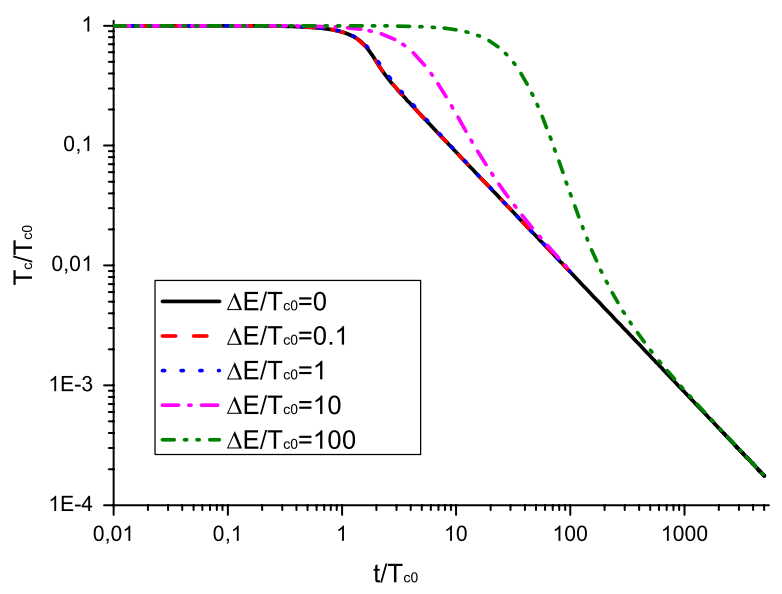

Figure 9. Graph of $T_{\mathrm{c}} / T_{\mathrm{c} 0}$ and $\Delta / T_{\mathrm{c} 0}$ as a function of $t / T_{\mathrm{c} 0}$. For $t \ll \Delta E \ll T_{\mathrm{c} 0}$, the critical temperature decreases slightly with the interlayer coupling. For $T_{\mathrm{c} 0} \ll t \lesssim \Delta E$, the critical temperature decreases with the interlayer coupling differently from in the case $T_{\mathrm{c} 0} \ll \Delta E \ll t$.

3.2.1. Superconducting critical temperature as a function of $t$ and $\Delta E$. We use the Green function (24) and the selfconsistency equation (3) to determine the superconducting critical temperature:

$$
\begin{aligned}
\ln \left(\frac{T_{\mathrm{c}}}{T_{\mathrm{c} 0}}\right)= & -\frac{t^{2}}{\Delta E^{2}+4 t^{2}}[2 \gamma+4 \ln (2) \\
& +\Psi\left(\frac{1}{2}-\frac{i \sqrt{\Delta E^{2}+4 t^{2}}}{4 \pi T_{\mathrm{c}}}\right) \\
& \left.+\Psi\left(\frac{1}{2}+\frac{i \sqrt{\Delta E^{2}+4 t^{2}}}{4 \pi T_{\mathrm{c}}}\right)\right] .
\end{aligned}
$$

The variation of the critical temperature is presented in figure 9 for different values of $\Delta E$. The variation of the critical temperature is described by three energy scales: $t, \Delta E$ and $T_{\mathrm{c} 0}$. For small interlayer coupling $t \ll$ $\Delta E \ll T_{\mathrm{c} 0}$, the critical temperature varies as $\frac{T_{\mathrm{c}}-T_{\mathrm{c} 0}}{T_{\mathrm{c} 0}}=$ $-\frac{1}{8 \pi^{2}}\left(\frac{t}{T_{\mathrm{c} 0}}\right)^{2}\left(7 \zeta(3)-\frac{31}{\pi^{2}} \zeta(5)\left(\frac{\Delta E}{T_{\mathrm{c} 0}}\right)^{2}\right)$. If the interlayer coupling is bigger than the energy shift, $\Delta E \ll t \ll$ $T_{\mathrm{c} 0}$, then $\frac{T_{\mathrm{c}}-T_{\mathrm{c} 0}}{T_{\mathrm{c} 0}}=-\frac{7 \zeta(3)}{8 \pi^{2}}\left(\frac{t}{T_{\mathrm{c} 0}}\right)^{2}$, which corresponds to the classical case. We note that the critical temperature decreases slower in the presence of an energy shift $\Delta E$ in the regime of small interlayer coupling. For the strong energy shift in the small interlayer coupling regime, $t \ll$ $T_{\mathrm{c} 0} \ll \Delta E$, the critical temperature varies as $\frac{T_{\mathrm{c}}-T_{\mathrm{c} 0}}{T_{\mathrm{c} 0}}=$ $-\left(\frac{t / T_{\mathrm{c} 0}}{\Delta E / T_{\mathrm{c} 0}}\right)^{2}\left[2 \gamma+\ln \left(\frac{\Delta E}{4 \pi^{2}}\right)\right]$. In this case, the variation is slow because of the decrease of the effective interlayer coupling. The mismatch of the Fermi surface induces more difficult tunneling of the Cooper pairs between the $S$ and the $\mathrm{N}$ layers. For high interlayer coupling, the critical temperature $T_{\mathrm{c}}$ decreases. For $T_{\mathrm{c} 0} \ll t \simeq \Delta E$, the variation of temperature is written as $\frac{T_{\mathrm{c}}}{T_{\mathrm{c} 0}} \simeq \frac{\pi \mathrm{e}^{-\gamma}}{\sqrt{\Delta E^{2}+4 t^{2}}}$. The effective interlayer coupling is bigger than the real interlayer coupling.

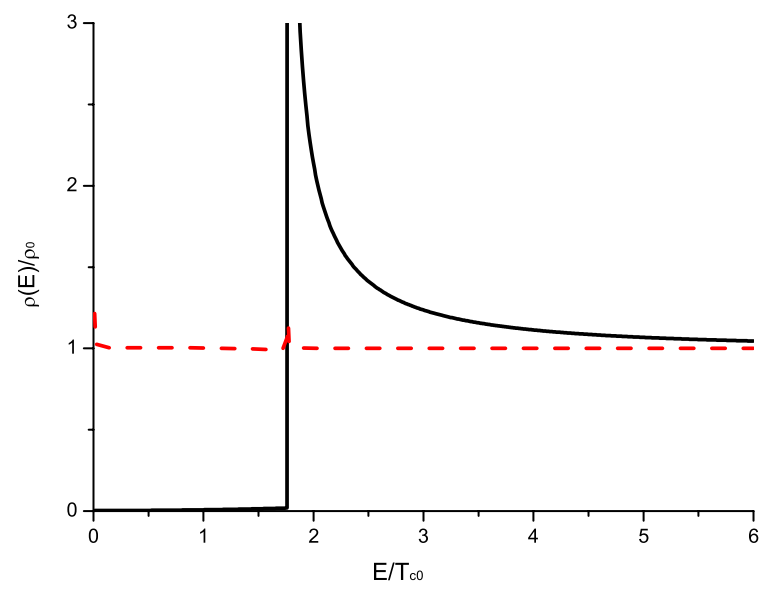

Figure 10. Graph of density of states $\rho(E) / \rho_{0}$ in the $\mathrm{S}$ layer (solid line) and in the $\mathrm{N}$ layer (dashed line) for $t=0.1 T_{\mathrm{c} 0}$ and an energy shift $\Delta E / T_{\mathrm{c} 0}=0.1 T_{\mathrm{c} 0}$.

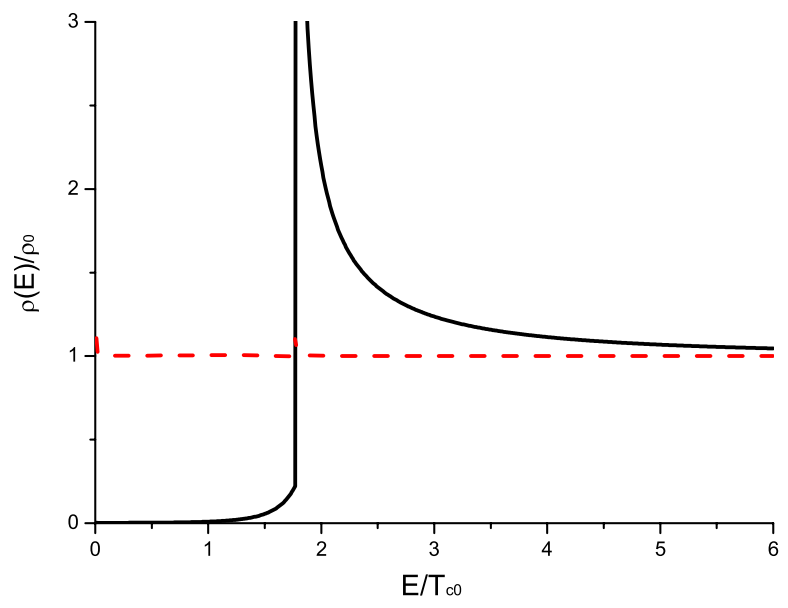

Figure 11. Graph of density of states $\rho(E) / \rho_{0}$ in the S layer (solid line) and in the $\mathrm{N}$ layer (dashed line) for $t=0.1 T_{\mathrm{c} 0}$ and an energy shift $\Delta E / T_{\mathrm{c} 0}=T_{\mathrm{c} 0}$.

For $T_{\mathrm{c} 0} \ll \Delta E \ll t$ and $\Delta E \ll T_{\mathrm{c} 0} \ll t$, the results are the same as described in [32]. As in the small interlayer coupling regime, the mismatch between the Fermi surface weakens the proximity effect.

3.2.2. Density of states in the $S$ and the $N$ layers as a function of t and $\Delta E$. An energy shift between the $\mathrm{S}$ and $\mathrm{N}$ electronic spectrum influences the density of states of the $\mathrm{S}$ and $\mathrm{N}$ layers. From the relation (15), we can determine the density of states for different configurations. The energy shift must influence the density of states by affecting the number of available states in the $\mathrm{S} / \mathrm{N}$ structure. In the small interlayer coupling limit, $t \ll T_{\mathrm{c} 0}$ approximation, for energy shifts $\Delta E<\Delta(0)$ the DOS in $\mathrm{S}$ and $\mathrm{N}$ layers are nearly the same as in a single $\mathrm{N}$ layer, see figures 10 and 11 . In the case where the energy shift is higher than the superconducting gap, $\Delta E>\Delta$, there appear DOS peaks for energy $E$ around $\frac{\Delta E}{2}$. (See figure 12). These peaks may correspond to the tunneling of particles between the $\mathrm{S}$ and the $\mathrm{N}$ layer that occurs at these energies. 


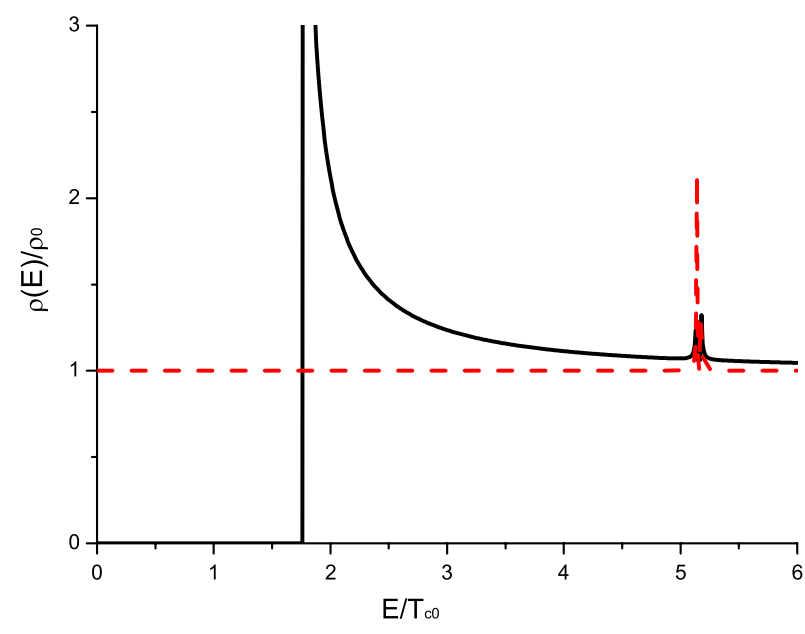

Figure 12. Graph of density of states $\rho(E) / \rho_{0}$ in the $\mathrm{S}$ layer (solid line) and in the $\mathrm{N}$ layer (dashed line) for $t=0.1 T_{\mathrm{c} 0}$ and an energy shift $\Delta E / T_{\mathrm{c} 0}=10 T_{\mathrm{c} 0}$.

In the case of high energy shift and high interlayer coupling, $t=100 T_{\mathrm{c} 0}$ and $\Delta E=100 T_{\mathrm{c} 0}$, the density of states may be influenced by the formation of a hybrid level between the two layers. In figure 13, the DOS varies in a complex manner with the energy. We note that the DOS in the two layers are almost the same. At high interlayer coupling, the $\mathrm{S} / \mathrm{N}$ bilayer can be considered as a single $\mathrm{S}$ layer with a superconducting constant divided by two [32].

As we have demonstrated, the properties of the bilayers strongly depend on the interlayer coupling and the mismatch between the $\mathrm{S}$ and $\mathrm{N}$ layers Fermi surfaces that produce energy shift between the electronic energy spectra. This energy shift makes the electronic transfer between the $\mathrm{S}$ and $\mathrm{N}$ layer more difficult. The presence of the energy shift may be observed in the peaks in the DOS of the S and N layers.

\subsection{Density of states in the $\mathrm{S} / \mathrm{N} / \ldots \mathrm{N}$ multilayer for weak interlayer coupling}

In this subsection we study the DOS in the $\mathrm{S} / \mathrm{N} / \ldots / \mathrm{N}$ multilayer in the weak coupled approximation $t \ll T_{\mathrm{c}}$. The dependence of the Gorkov Green function in the $\mathrm{S} / \mathrm{N} / \ldots / \mathrm{N}$ multilayer on the total number of layers $L$ is simpler in the case of weak interlayer coupling. This study may be relevant for clean quasi-bidimensional devices.

3.3.1. Critical temperature of the $\mathrm{S} / \mathrm{N} / \ldots \mathrm{N}$ multilayer. To find the properties of the $\mathrm{S}$ layer, we use the anomalous Green function (9). The arbitrary number of total number of layers in the $\mathrm{S} / \mathrm{N} / \ldots / \mathrm{N}$ multilayer makes it difficult to find the solution to the self-consistency equation in the general case. However, we can easily determine the behavior of the critical temperature in the case of a weak interlayer coupling $t \ll T_{\mathrm{c} 0}$. In this regime the transfer of the superconducting correlations in the adjacent $\mathrm{N}$ layers is highly limited and decreases fast. Consequently, the critical temperature of the $\mathrm{S}$ layer varies very little with the interlayer coupling. In the weak interlayer

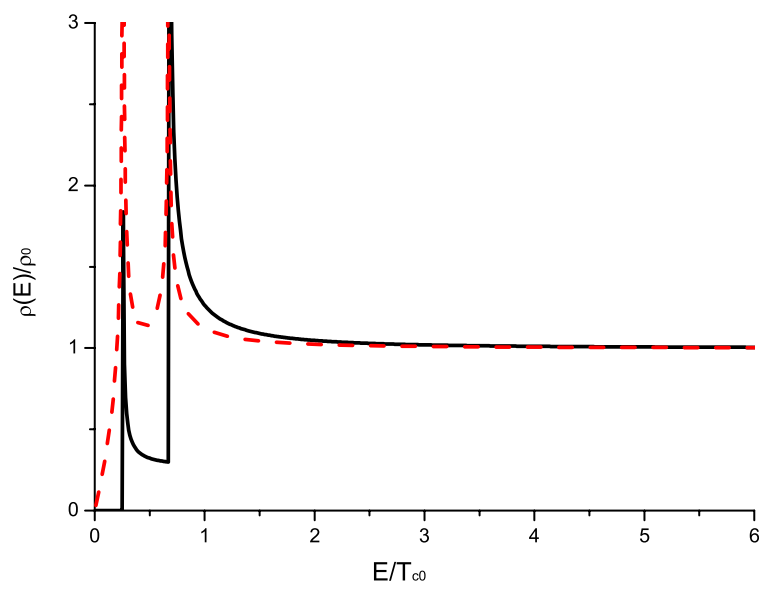

Figure 13. Graph of the density of states $\rho(E) / \rho_{0}$ in the $\mathrm{S}$ layer (solid line) and in the $\mathrm{N}$ layer (dashed line) for $t=100 T_{\mathrm{c} 0}$ and an energy shift $\Delta E / T_{\mathrm{c} 0}=100 T_{\mathrm{c} 0}$.

regime, $t \ll T_{\mathrm{c} 0}$, we may replace the hyperbolic sines in the Green function (9), by the expressions

$$
\begin{gathered}
a_{1}=\sinh (q) \simeq \frac{\left(\mathrm{i} \omega-\xi_{n}\right)}{2 t} \\
\widetilde{a}_{1}=\sinh (\widetilde{q}) \simeq-\frac{\left(\mathrm{i} \omega+\xi_{n}\right)}{2 t} .
\end{gathered}
$$

The anomalous Green function $F_{\downarrow, \uparrow}^{\dagger 0,0}$ can be written as

$$
\begin{aligned}
F_{\downarrow, \uparrow}^{\dagger 0,0}= & \frac{\Delta_{0}^{*}}{\left(\omega^{2}+\xi_{\mathrm{s}}^{2}+\left|\Delta_{0}\right|^{2}\right)} \\
& \times\left[1-\frac{2 t^{2}}{\left(\omega^{2}+\xi_{\mathrm{s}}^{2}+\left|\Delta_{0}\right|^{2}\right)} \frac{\left(\omega^{2}-\xi_{\mathrm{s}}^{2}\right)}{\omega^{2}+\xi_{\mathrm{s}}^{2}}\right. \\
& -\frac{t^{4}}{\left(\omega^{2}+\xi_{\mathrm{s}}^{2}+\left|\Delta_{0}\right|^{2}\right)\left(\omega^{2}+\xi_{\mathrm{s}}^{2}\right)} \\
& \left.\times\left(1-\frac{4}{\left(\omega^{2}+\xi_{\mathrm{s}}^{2}+\left|\Delta_{0}\right|^{2}\right)} \frac{\left(\omega^{2}-\xi_{\mathrm{s}}^{2}\right)^{2}}{\left(\omega^{2}+\xi_{\mathrm{s}}^{2}\right)}\right)\right] .
\end{aligned}
$$

From the self-consistency equations (3), the critical temperature can be written as $\frac{T_{\mathrm{c}}}{T_{\mathrm{c} 0}}=1-\frac{7}{8} \frac{\zeta(3)}{\pi^{2}}\left(\frac{t}{T_{\mathrm{c} 0}}\right)^{2}$. This variation is the same as in the $\mathrm{S} / \mathrm{N}$ bilayer and the $\mathrm{S} / \mathrm{N} / \mathrm{N}$ trilayer in the small interlayer coupling regime. In this regime, only the nearest $\mathrm{N}$ layer is important and affects the superconducting properties. The superconducting gap at zero temperature reads $\frac{\Delta(0)-\Delta}{\Delta}=\frac{4 t^{4}}{|\Delta(0)|^{4}} \ln \left(\frac{t}{|\Delta(0)|}\right)$. This variation is typical for the weak interlayer coupling regime where the logarithm dependence appears [33]. The superconducting gap is affected only by the nearest $\mathrm{N}$ layer.

3.3.2. Density of states in the last metal layer of the $\mathrm{S} / \mathrm{N} / \ldots \mathrm{N}$ multilayer. We have determined the Green functions in the $\mathrm{S}$ and in the last metal layer. We are first interested in the case where the $\mathrm{S}$ and the $\mathrm{N}$ layers Fermi surfaces are perfectly matching. For small interlayer coupling, 
$t \ll T_{\mathrm{c} 0}$, the properties of the $\mathrm{S}$ layer are the same as those of the $\mathrm{S} / \mathrm{N}$ bilayer [32]. The normal Green function of the last $\mathrm{N}$ layer $G_{\uparrow, \uparrow}^{L, L}$ is the same as in a single metal layer. Then, to take into account the transmission of the superconducting correlations in this approximation, we consider that the transmission of the superconducting correlations is highly reduced, which implies that the superconducting gap in the last $\mathrm{N}$ layer is small. We make an expansion on $\Delta^{2}$ of the normal Green function. From this expansion, we will study the DOS in the last metal layer in the case of a perfect match and a mismatch between the $\mathrm{S}$ and $\mathrm{N}$ layers Fermi surfaces.

\section{Perfect match between the $\mathrm{N}$ and $\mathrm{S}$ layer Fermi surfaces}

With a perfect match between the $\mathrm{S}$ and $\mathrm{N}$ layer Fermi surfaces, the electronic spectra in the $\mathrm{S}$ and the $\mathrm{N}$ layers are the same, $\xi_{\mathrm{s}}=\xi_{n}$. The Green function in (7), expanded in $\Delta^{2}$, can be written in the approximation $t \ll T_{\mathrm{c} 0}$ as

$G_{\uparrow \uparrow}^{L, L}=\frac{1}{\mathrm{i} \omega-\xi_{\mathrm{s}}}+\Delta_{0}^{2} \frac{1}{\left(\mathrm{i} \omega-\xi_{\mathrm{s}}\right)^{2}\left(\mathrm{i} \omega+\xi_{\mathrm{s}}\right)}\left(\frac{t}{\mathrm{i} \omega-\xi_{\mathrm{s}}}\right)^{2 L}$

where the hyperbolic sines are replaced by the expression (27). We note that the superconducting correlations depend directly on the interlayer coupling to the power $2 L$. In this case, the superconducting correlations for a large number of layers are negligible. From the relation (15), we determine the correction to the DOS of the last metal layer related to the superconducting correlations:

$$
\Delta \rho=\frac{\rho^{L}(E)-\rho_{0}}{\rho_{0}}=\frac{\Delta_{0}^{2}}{2 E^{2}}\left(\frac{t}{2 E}\right)^{2 L}
$$

which is valid in the approximation $E>t$. The superconducting correlations affect the density of states at the power $2 L$. This dependence is logical because the superconducting correlations concern two electrons transmitted through $L$ layers. As expected, the influence of the $S$ layer on the last metal layers is very small for a large number of layers $L$ in weakly coupled multilayers.

\section{Mismatch between the $\mathrm{N}$ and $\mathrm{S}$ layer Fermi surfaces}

The Green function in (7), expanded in $\Delta^{2}$, can be written in the approximation $t \ll T_{\mathrm{c} 0}$ as

$$
G_{\uparrow \uparrow}^{L, L}=\frac{1}{\left(\mathrm{i} \omega-\xi_{n}\right)}+\frac{\Delta_{0}^{2}}{\left(\mathrm{i} \omega+\xi_{\mathrm{s}}\right)\left(\mathrm{i} \omega-\xi_{\mathrm{s}}\right)^{2}}\left(\frac{t}{\mathrm{i} \omega-\xi_{n}}\right)^{2 L}
$$

where the hyperbolic sines are replaced by the expression (27). The superconducting properties merge near the $S$ layer Fermi momentum. Due to the difference between the $\mathrm{N}$ and $\mathrm{S}$ layers electronic band structure, a mismatch between the $\mathrm{S}$ and $\mathrm{N}$ layers Fermi surfaces can occur. In the approximation of parabolic dispersion, this mismatch of Fermi surfaces can manifest as an energy shift $\Delta E$ and a different effective mass between the $\mathrm{S}$ and $\mathrm{N}$ layer electronic spectra. There are two major contributions to the $\mathrm{S} / \mathrm{N} / \ldots / \mathrm{N}$ multilayer properties. The first is the contribution of the electronic state near the superconductor layer's Fermi surface (see figure 14(b)). In this case, the integration in the relation (15) is made near the $\mathrm{S}$ layer Fermi surface with the $\mathrm{N}$ layer electronic spectrum strongly shifted. The second contribution originates from the electronic states near the normal metal Fermi surface (see figure 14(a)). In this situation, the integration in the relation (15) is made near the $\mathrm{N}$ layer Fermi surface with the S layer electronic spectrum strongly shifted. We evaluate the contribution of the states near the $\mathrm{N}$ and $\mathrm{S}$ layer Fermi surfaces, in the case of an arbitrary number of layers $L$, to determine the greater contribution and then determine which layers influence the properties of the multilayer.

\section{Contribution of the states near the $\mathrm{S}$ layer Fermi surfaces}

In this situation, the integration can be made by considering the $\mathrm{S}$ and $\mathrm{N}$ layer electronic spectra in the following form:

$$
\begin{aligned}
\xi_{\mathrm{s}} & =\frac{p^{2}}{2 m_{\mathrm{S}}}-E_{\mathrm{F}} \simeq v_{\mathrm{F}_{\mathrm{S}}}\left(p-p_{\mathrm{F}_{\mathrm{s}}}\right) \\
\xi_{n} & =\frac{p^{2}}{2 m_{n}}-E_{\mathrm{F}} \simeq \frac{p_{\mathrm{F}_{\mathrm{S}}}^{2}}{2 m_{n}}-E_{\mathrm{F}}=\frac{p_{\mathrm{F}_{\mathrm{S}}}^{2}-p_{\mathrm{F}_{n}}^{2}}{2 m_{n}} \\
& =\Delta E \gg T_{\mathrm{c} 0}
\end{aligned}
$$

where $v_{\mathrm{F}_{\mathrm{S}}(n)}$ is the Fermi velocity in the superconductor (normal metal), $p_{\mathrm{F}_{\mathrm{S}(n)}}$ the Fermi impulsion in the superconductor (normal metal) and $\Delta E$ is the energy difference between the $\mathrm{N}$ and $\mathrm{S}$ layer electronic spectra for an impulsion equal to $p_{\mathrm{F}_{\mathrm{S}}}$. The integral $\int d p$ becomes $\int \frac{\mathrm{d} \xi_{\mathrm{s}}}{v_{\mathrm{F}_{\mathrm{s}}}}$. The correction to the density of states can be written as:

$$
\Delta \rho=\frac{\rho^{L}(E)-\rho_{0}}{\rho_{0}}=\frac{\Delta^{2}}{2 E^{2}}\left(\frac{t^{2}}{4 E^{2}+(\Delta E)^{2}}\right)^{L}
$$

which is valid in the limit $E>t$. The superconducting correlations decrease as a function of $t^{2 L}$. Moreover, the energy shift appears directly in the density of states. In the regime $\Delta E \gg T_{\mathrm{c} 0}$, the correction to the density of states can be written as $\Delta \rho=\frac{\Delta^{2}}{2 E^{2}}\left(\frac{t}{\Delta E}\right)^{2 L}$, which is very small.

\section{Contribution of the state near the $\mathrm{N}$ layer Fermi surface}

In this situation, we consider the state near the $\mathrm{N}$ layer Fermi surface, the energy spectra can be written in the following way

$$
\begin{aligned}
\xi_{\mathrm{s}} \simeq \frac{p_{\mathrm{F}_{n}}^{2}}{2 m_{\mathrm{S}}}-E_{\mathrm{F}} & =-\frac{m_{n}}{m_{\mathrm{S}}} \Delta E=\xi_{n}+\widetilde{\Delta E} \gg T_{\mathrm{c}} \\
\xi_{n} & =v_{\mathrm{F}_{n}}\left(p-p_{\mathrm{F}_{\mathrm{S}}}\right) .
\end{aligned}
$$

The correction to the density of states can be written as

$$
\Delta \rho=\frac{\Delta_{0}^{2}}{2 E^{2}}\left(\frac{t^{2}}{4 E^{2}+(\widetilde{\Delta E})^{2}}\right)^{L}
$$

valid in the limit $E>t$. The superconducting correlations decrease as a function of $t^{2 L}$. In the approximation of strong energy shift $\widetilde{\Delta E} \gg T_{\mathrm{c} 0}$, the correction to the density of states can be written as $\Delta \rho=\frac{\Delta^{2}}{2 E^{2}}\left(\frac{t}{\widetilde{\Delta E}}\right)^{2 L}$, which is very small. However, the contribution depends on the values of both $\Delta E$ and $\widetilde{\Delta E}$. The energy shift $\widetilde{\Delta E}$ depends on the effective mass in the metal and in the superconductor.

3.3.3. Conclusion. The superconducting correlations strongly decrease in the last $\mathrm{N}$ layer as a function of $t^{2 L}$. As 
expected, the proximity effect weakens with the number of layers. In the case of a mismatch between the $\mathrm{S}$ and $\mathrm{N}$ layer Fermi surfaces, the density of states in the last $\mathrm{N}$ layer depends on the contribution of the state near the $\mathrm{S}$ or $\mathrm{N}$ layer Fermi surface. These corrections depend on the effective mass ratio $m_{n} / m_{\mathrm{s}}$. If $m_{n}<m_{\mathrm{s}}$ then the contribution is greater for the state near the $\mathrm{N}$ layer Fermi surface. This means that the properties of the multilayer are driven by the states near the $\mathrm{N}$ layer Fermi surface. If $m_{n}>m_{\mathrm{s}}$ then the contribution is greater for the states near the $\mathrm{S}$ layer Fermi surface. This means that the properties of the multilayer are driven by the states near the $\mathrm{S}$ layer Fermi surface. If $m_{n}=m_{\mathrm{s}}$, the properties are driven by both the states near the $\mathrm{S}$ and $\mathrm{N}$ layer Fermi surfaces. This study demonstrates the crucial role of the intrinsic properties of the layers composing a $\mathrm{S} / \mathrm{N} / \ldots / \mathrm{N}$ multilayer. The difference of effective mass can determine which layers influence the properties of the $\mathrm{S} / \mathrm{N} / \ldots / \mathrm{N}$ multilayer. A study of this effect for strong interlayer coupling could determine the role of each Fermi surface.

\section{Josephson current and $0-\pi$ transition phase in the $S / N / \ldots / N / S$ junction}

The S/N/S hybrid trilayer has been extensively studied since the discovery of the Josephson effect [15, 30, 31, 58]. We apply the model which has been described in the first part and add an arbitrary number of metal layers between the two superconducting layers. First we determine the superconducting gap in the 0 and the $\pi$ states of the first superconducting layer in the case of small interlayer coupling. The $0(\pi)$ state occurs when the two S layers support the same (opposite) signs of the order parameter [59]. We deduce the free energy of the junction and the Josephson critical current. The superconducting layers are labeled $n=0$ and $n=L$. The normal metal layers between the two $S$ layers are labeled $n=1$ to $L-1$ (see figure $1(\mathrm{~b})$ ). We study the influence of the metallic spectrum on the global properties of the junction.

\subsection{Superconducting gap in the $\mathrm{S} / \mathrm{N} / \ldots \mathrm{N} / \mathrm{S}$ junction}

To find the superconducting gap of the $\mathrm{S} / \mathrm{N} / \ldots / \mathrm{N} / \mathrm{S}$ Josephson junction, we use the Green function presented in (4). If we consider a weak interlayer coupling $t \ll T_{\mathrm{c} 0}$, the anomalous Green function (14) of the first superconducting layer can be written as

$$
\begin{aligned}
F_{\downarrow, \uparrow}^{\dagger 0,0}= & \frac{\Delta_{0}^{*}}{\left(\omega^{2}+\xi_{\mathrm{s}}^{2}+\Delta_{0}^{2}\right)} \\
& +\frac{\Delta_{L}^{*}\left(\omega^{2}+\xi_{n}^{2}\right)\left(\omega^{2}+\xi_{\mathrm{s}}^{2}-\Delta_{0}^{2}\right)}{\left(\omega^{2}+\xi_{\mathrm{s}}^{2}+\Delta_{0}^{2}\right)^{2}\left(\omega^{2}+\xi_{\mathrm{s}}^{2}+\Delta_{L}^{2}\right)} \\
& \times\left(\frac{t^{2}}{\omega^{2}+\xi_{n}^{2}}\right)^{L}
\end{aligned}
$$

where we keep the terms to the power $2 L$ which change the sign with the $0-\pi$ phase transition. We see that the interlayer term directly depends on the metallic spectrum.
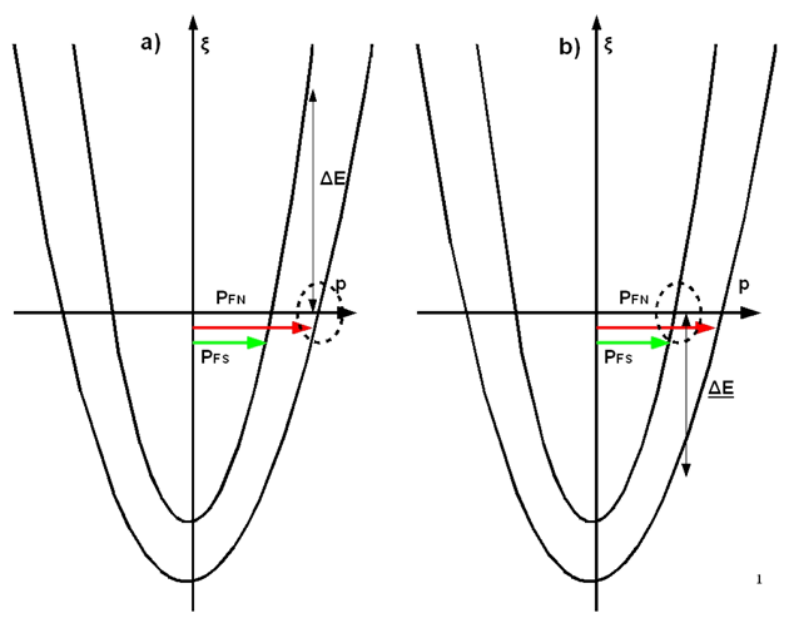

Figure 14. Electronic spectra in the $\mathrm{S}$ and $\mathrm{N}$ layers in the two integration cases. In the case (a), the integration is near the metallic Fermi surface. In the case (b), the integration is near the superconducting Fermi surface. The dashed circle shows the place where the integration is made.

This means that the proximity effect in the S/N/S multilayer is highly affected by the form of the electronic spectra of the metallic layers. When the junction is in the phase $0(\pi)\left(\Delta_{0}^{(*)}= \pm \Delta_{L}^{(*)}\right)$, the function (36) can be written as:

$$
\begin{aligned}
F_{\downarrow, \uparrow}^{\dagger 0,0}= & \frac{\Delta^{0(\pi)^{*}}}{\left(\omega^{2}+\xi_{\mathrm{s}}^{2}+\left|\Delta^{0(\pi)}\right|^{2}\right)} \\
& \pm \frac{\Delta^{0(\pi)^{*}}\left(\omega^{2}+\xi_{n}^{2}\right)\left(\omega^{2}+\xi_{\mathrm{s}}^{2}-\left|\Delta^{0(\pi)}\right|^{2}\right)}{\left(\omega^{2}+\xi_{\mathrm{s}}^{2}+\left|\Delta^{0(\pi)}\right|^{2}\right)^{3}} \\
& \times\left(\frac{t^{2}}{\omega^{2}+\xi_{n}^{2}}\right)^{L},
\end{aligned}
$$

where $\Delta^{0(\pi)}$ is the superconducting gap when the junction is in the $0(\pi)$-phase. The self-consistency equation in the 0 or the $\pi$ phase can be written as:

$$
\begin{aligned}
& \ln \left(\frac{\Delta}{\Delta^{0(\pi)}}\right)=\pi T \\
& \quad \times \sum_{\omega} \int \mathrm{d} \xi_{\mathrm{s}}\left[\mp \frac{\left(\omega^{2}+\xi_{n}^{2}\right)\left(\omega^{2}+\xi_{\mathrm{s}}^{2}-\left|\Delta^{0(\pi)}\right|^{2}\right)}{\left(\omega^{2}+\xi_{\mathrm{s}}^{2}+\left|\Delta^{0(\pi)}\right|^{2}\right)^{3}}\right. \\
& \left.\quad \times\left(\frac{t^{2}}{\omega^{2}+\xi_{n}^{2}}\right)^{L}-\frac{1}{\left(\omega^{2}+\xi_{\mathrm{s}}^{2}+|\Delta|^{2}\right)}\right]
\end{aligned}
$$

where we note that the left hand side term is evaluated at zero temperature. For a weak interlayer coupling, we can consider that the difference between the superconducting gap in the 0 and $\pi$ phase is the same at zero temperature as at finite but very low temperature. The difference $\Delta^{0}-\Delta^{\pi}$ is very small in the approximation $t \ll T_{\mathrm{c} 0}$. This difference evolves as a function of $t^{2 L}$. The relation between $\Delta^{0}$ and $\Delta^{\pi}$ can be 
written as:

$$
\begin{aligned}
& \ln \left(\frac{\Delta^{0}}{\Delta^{\pi}}\right) \\
& =\pi T \sum_{\omega} \int \mathrm{d} \xi_{\mathrm{s}}\left[\frac{\left(\omega^{2}+\xi_{n}^{2}\right)\left(\omega^{2}+\xi_{\mathrm{s}}^{2}-|\Delta|^{2}\right)}{\left(\omega^{2}+\xi_{\mathrm{s}}^{2}+|\Delta|^{2}\right)^{3}}\right. \\
& \left.\quad \times\left(\frac{t^{2}}{\omega^{2}+\xi_{n}^{2}}\right)^{L}\right]
\end{aligned}
$$

where we consider $\Delta$ is an average superconducting gap $\frac{\Delta_{0}+\Delta_{\pi}}{2}$. From the self-consistency equation (39), we can find the difference $\Delta^{0}-\Delta^{\pi}$, which in the case of the multilayer is:

$$
\begin{aligned}
& \frac{\Delta^{0}-\Delta^{\pi}}{\Delta^{\pi}} \\
& =\pi T \sum_{\omega} \int_{0}^{+\infty}\left[\frac{t^{2 L}\left(\omega^{2}+\xi_{\mathrm{s}}^{2}-\Delta^{2}\right)}{\left(\omega^{2}+\xi_{n}^{2}\right)^{L-1}\left(\omega^{2}+\xi_{\mathrm{s}}^{2}+\Delta^{2}\right)^{3}}\right] \mathrm{d} \xi_{\mathrm{s}} .
\end{aligned}
$$

At low temperature, this difference is negative, which implies a superconducting gap in the 0 state smaller than in the $\pi$ state. These difference depends on the number of layers and then on the thickness of the junction. With a perfect mismatch of the $S$ and $\mathrm{N}$ layer Fermi surfaces, then the electronic spectra in the $\mathrm{S}$ and $\mathrm{N}$ layers can be considered as equal, $\xi_{n}=\xi_{\mathrm{s}}$, and the difference of the superconducting gap is equal to

$$
\frac{\Delta^{0}-\Delta^{\pi}}{\Delta^{\pi}}=\frac{-T^{4} \zeta(2 L-3)}{\Delta^{4} 2^{L-1} \pi^{2 L-4}(L-2) !} \frac{2^{2 L-3}-1}{2^{2 L-3}}\left(\frac{t}{T}\right)^{2 L}
$$

where we see that this difference is negative at low temperature, which implies a superconducting gap higher in the $\pi$ phase than in the 0 phase. This difference is non-intuitive, because the 0 state is more stable than the $\pi$-state. We determine the free energy of the system in section 4.2. In the case of a mismatch between the $\mathrm{S}$ and $\mathrm{N}$ layers, the electronic spectra in the $\mathrm{S}$ and $\mathrm{N}$ layers are shifted.

4.1.1. Contribution of the states near the $S$ layer Fermi surface. We have a $\mathrm{N}$ layer spectrum shifted from the $\mathrm{S}$ layer spectrum. With the conditions (32), we set $\xi_{n}=\Delta E \gg T_{\mathrm{c}}$. The difference of the superconducting gaps (40) becomes:

$$
\frac{\Delta^{0}-\Delta^{\pi}}{\Delta^{\pi}}=\left(\frac{t}{\Delta E}\right)^{2 L} \frac{\Delta E^{2}\left(56 \zeta(3) \pi^{2} T_{\mathrm{c}}^{2}-31 \Delta^{2} \zeta(5)\right)}{32 \pi^{4} T_{\mathrm{c}}^{4}}
$$

after expansion in $\Delta$. We note that the difference $\Delta^{0}-\Delta^{\pi}$ is positive whatever the superconducting gap. This means that the contribution of the state near the S layer Fermi surface implies a superconducting gap greater in the 0 phase than in the $\pi$-phase. This difference decays rapidly with the energy shift between the $\mathrm{S}$ and $\mathrm{N}$ state electronic spectrum. At zero temperature, this difference vanishes.

4.1.2. Contribution of the state near the $N$ layer Fermi surface. From the conditions (34), we have $\xi_{\mathrm{s}}=-\frac{m_{n}}{m_{\mathrm{S}}} \Delta E \gg$
$T$. The difference between the superconducting gaps (40) becomes:

$$
\begin{aligned}
\frac{\Delta^{0}-\Delta^{\pi}}{\Delta^{\pi}}= & \frac{2^{2 L-3}-1}{2^{2 L-3}} \frac{\zeta(2 L-3) T^{4}}{\pi^{2 L-4} 2^{L-3}(L-2) !} \\
& \times \frac{\left(\left(\frac{m_{n}}{m_{\mathrm{S}}} \Delta E\right)^{2}-\Delta^{2}\right)}{\left(\left(\frac{m_{n}}{m_{\mathrm{S}}} \Delta E\right)^{2}+\Delta^{2}\right)^{3}}\left(\frac{t}{T}\right)^{2 L}
\end{aligned}
$$

in the case $\Delta \gg T$ and $t \ll T$. We note that at low temperature, the difference $\Delta^{0}-\Delta^{\pi}$ is negative in the case where $\frac{m_{n}}{m_{\mathrm{S}}} \Delta E$ is smaller than $\Delta$. In the case of a large energy shift $\frac{m_{n}}{m_{\mathrm{S}}} \Delta E \gg \Delta$, the difference $\Delta^{0}-\Delta^{\pi}$ is always positive. The influence of the states near the metallic Fermi surface can imply an inversion in the superconducting gap in the 0 and $\pi$ phase.

4.1.3. Conclusion. We note that this difference is always proportional to the interlayer coupling $t$ to the power $2 L$. As $t \ll T_{\mathrm{c} 0}$, this difference is rapidly decreasing with the thickness of the junction. At low temperature, where $T>t$ in the approximation $t \ll T_{\mathrm{c} 0}$, the two contributions are equivalent. For higher temperature, we see that the contribution of the states near the $\mathrm{S}$ layer Fermi surface is greater. This behavior seems to be coherent because the proximity effect and the superconducting correlations are strongly supported by the $\mathrm{S}$ layer. The superconducting state induced in the adjacent $\mathrm{N}$ layers may play a role at low temperature. These states disappear at higher temperature.

\subsection{Critical current in the $\mathrm{S} / \mathrm{N} / \ldots \mathrm{N} / \mathrm{S}$ junction}

At all temperatures, the free energy in the $\mathrm{S} / \mathrm{N} / \ldots / \mathrm{N} / \mathrm{S}$ junction between the normal and the superconducting state is determined by the relation [57]:

$$
F_{\mathrm{s}}-F_{n}=\int_{0}^{\Delta(0)}\left(\frac{\partial\left(\frac{1}{\lambda}\right)}{\partial \Delta} \Delta^{2}\right) \mathrm{d} \Delta .
$$

We can calculate the free energy in the case of weak interlayer coupling:

$$
\begin{aligned}
F_{\pi}- & F_{0}=4 t^{2 L} N(0) \Delta^{2} T \sum_{\omega} \int_{0}^{+\infty} \\
\times & {\left[\frac{1}{\left(\omega^{2}+\xi_{n}^{2}\right)^{L-1}\left(\omega^{2}+\xi_{\mathrm{s}}^{2}+\Delta^{2}\right)^{2}}\right] \mathrm{d} \xi_{\mathrm{s}}>0 }
\end{aligned}
$$

where we see that the free energy of the $\pi$ state is higher than the free energy in the 0 state. The $\pi$ state cannot appear in the $\mathrm{S} / \mathrm{N} / \ldots / \mathrm{N} / \mathrm{S}$ junction. In the case where $\xi_{n}=\xi_{\mathrm{s}}$, with the free energy difference and the relation [18], the Josephson critical current can be written as

$$
\begin{aligned}
I_{\mathrm{c}}= & \frac{\mathrm{e}}{\hbar} \frac{4 \pi \rho(0)(2 L-4) !}{((L-2) !)^{2}} \\
& \times \frac{\left(2^{(2 L-3)}-1\right)}{\Delta^{2} 2^{2 L-3}(2 \pi)^{2 L-3}} \zeta(2 L-3) T^{4} \mathrm{e}^{-\frac{x}{\xi}}
\end{aligned}
$$


where $\xi_{\mathrm{c}}$ is the interlayer superconducting coherence length, $x=L . a$ is the length of the $\mathrm{N}$ layer and $a$ is the interlayer distance. Here $\xi_{\mathrm{c}}$ can be written as

$$
\xi_{\mathrm{c}}=\frac{a}{2 \ln \left(\frac{T}{t}\right)} .
$$

We have $\xi_{\mathrm{c}} \longrightarrow 0$ if $T \longrightarrow \infty$. Moreover, we have $\xi \longrightarrow$ $\infty$ if $T \longrightarrow t$. Our approach is valid for $T \gg t$ because of the expansion in $t$. This limitation could be compensated by considering terms of higher order in $t$. We note an exponential decrease of the Josephson current in the metallic layers. This effect is characteristic of the decrease of the superconducting correlations in the metals established by superconductivity theory [41].

4.2.1. Contributions of the states near the $S$ layer Fermi surface $\xi_{\mathrm{s}} \ll \xi_{n}$. Using the relations (32), the critical Josephson current can be written as:

$$
I_{\mathrm{c}}=\frac{\mathrm{e}}{\hbar} \frac{\rho(0)(\Delta E)^{2} \Delta^{2}}{\pi^{2} T^{2}} \frac{7}{4} \zeta(3)\left(\frac{t}{\Delta E}\right)^{2 L} .
$$

The critical current is inversely proportional to the energy shift in the power $2 L$. The Josephson critical current is negligible and depends on the $\mathrm{N}$ layer characteristics. Near the $\mathrm{S}$ layer Fermi surface, the more general limit is the energy shift between the $\mathrm{S}$ and $\mathrm{N}$ layer electronic spectra.

4.2.2. Contributions of the states near the $N$ layer Fermi surface $\xi_{n} \ll \xi_{s}$. From the conditions (34), the critical Josephson current can be written as:

$$
\begin{aligned}
I_{\mathrm{c}}= & \frac{\mathrm{e}}{\hbar} \frac{\rho(0) \Delta^{2} \zeta(2 L-3) T^{4}}{2^{L-1} \pi^{2 L-4}(L-2) !\left(\left(\frac{m_{n}}{m_{\mathrm{S}}} \Delta E\right)^{2}+\Delta^{2}\right)^{2}} \\
& \times \frac{2^{2 L-3}-1}{2^{2 L-3}}\left(\frac{t}{T}\right)^{2 L} .
\end{aligned}
$$

In this Fermi surface configuration, the critical current decreases with temperature as a power law. This contribution may be relevant at low temperature. The divergence in temperature is due to the approximation $t \ll T$ and may be compensated by considering higher order terms in $t$.

4.2.3. Conclusion. For a high number of layers, at low temperature or for $\Delta E>t$, the highest contribution is for the state near the $\mathrm{N}$ layer Fermi surface. However, for high temperature or for a small number of layers, the highest contribution is for the state near the $\mathrm{S}$ layer Fermi surface. The Josephson current of a $\mathrm{S} / \mathrm{N} / \ldots / \mathrm{N} / \mathrm{S}$ junction depends explicitly on the number of metallic layers and on the energy shift $\Delta E$. We note that the critical current decreases faster with temperature for thick junctions [58].

\section{Conclusion}

We propose a solution to the Gorkov equations of a $\mathrm{S} / \mathrm{N}$ multilayered systems. With the Gorkov equation, we demonstrate the possibility to determine the properties of multilayered tunneling systems taking into account the superconducting correlations existing in these systems and a possible mismatch of the $\mathrm{S}$ and $\mathrm{N}$ layer Fermi surfaces.

The DOS in the $\mathrm{S} / \mathrm{N} / \ldots / \mathrm{N}$ system has a complex behavior that depends on the number of layers in the system. Then, the DOS of S/N/N trilayers exhibits three peaks which are the signature the superconducting gap in the $\mathrm{S}$ layer and induced a superconducting gap in the $\mathrm{N}$ layers by the proximity effect. A generalization of this approach may lead to the presence of $\mathrm{L}$ peaks in a $\mathrm{S} / \mathrm{N} / \ldots / \mathrm{N}$ multilayered structure composed by L layers. These peaks may be easier to observe if $t \approx \Delta$ at low temperatures because the induced superconducting gap in the $\mathrm{N}$ layers is proportional to $\frac{t^{2}}{\Delta}$. The correction to the superconducting correlation in the last $\mathrm{N}$ layer in the $\mathrm{S} / \mathrm{N} / \ldots / \mathrm{N}$ structure is proportional to $t^{2 L}$ in the tunneling approximation $t \ll T_{\mathrm{c}}$. As expected, the last $\mathrm{N}$ layer is weakly affected by the $\mathrm{S}$ layer. The density of states in such a system is gapless, as observed in S/N [45] and S/F [59] superlattices. In the $\mathrm{S} / \mathrm{N}$ case, the gapless character of the spectrum is due to the presence of an electron in the $\mathrm{N}$ layers that does not feel the superconducting pairing potential [45]. Nevertheless, in the case of a $\mathrm{S}$ and $\mathrm{N}$ layer Fermi surface mismatch, the density of states of the last $\mathrm{N}$ layer is influenced by the energy shift which exists between the $\mathrm{S}$ and $\mathrm{N}$ layer electronic spectra. Then, a difference between effective masses could change the dominant contribution of the $\mathrm{N}$ or $\mathrm{S}$ layer to the $\mathrm{S} / \mathrm{N} / \ldots / \mathrm{N}$ properties, such as DOS. This information helps to understand the properties of these multilayered systems. This contribution may be observed in epitaxial systems by STM methods.

The example of the superconducting gap in the 0 and the $\pi$ phase and the critical current in the S/N/ . . /N/S Josephson junction is another example of the influence of the energy shift mismatch and the long range superconducting correlation in multilayered systems. Unexpectedly, the superconducting gap in the $\pi$ phase is found to be higher than in the 0 phase. The $\pi$ phase is less stable than the 0 phase. These difference in the superconducting gaps and the logarithmic dependence of the coherence length are two signatures of the tunneling aspect of the Josephson junction. This dependence is directly related to the layered aspect of the Josephson junction in the tunneling approximation $t \ll T_{\mathrm{c}}$. Here, the Josephson junction has to be considered as a stack of alternating $\mathrm{N}$ independent layers. This discrete aspect in the construction of the Josephson junction may directly be the cause of this highly unusual dependence. Moreover, a possible mismatch between the $\mathrm{S}$ and $\mathrm{N}$ Fermi surfaces leads to complex behavior of the coherence length. The contribution of the $\mathrm{S}$ and $\mathrm{N}$ layer Fermi surfaces qualitatively changes the behavior of the critical current in multilayered Josephson junctions.

\section{Acknowledgments}

We are grateful to S Burdin and Y Gordeliy for helpful discussions. This work was supported, in part, by the French ANR program 'MASH' and the LabEX 'Amadeus' program. 


\section{Appendix. Gorkov Green function in S/N/ .../N multilayer and $\mathrm{S} / \mathrm{N} / \ldots$. . $/ \mathrm{S}$ junctions}

This appendix aims at evaluating the Gorkov functions in $\mathrm{S} / \mathrm{N} / \ldots$. . $\mathrm{N}$ multilayers and S/N/ .../N/S junctions.

\section{Propagation conditions}

These propagation conditions exist in the system of equations that describes the $\mathrm{S} / \mathrm{N} / \ldots / \mathrm{N}$ multilayers and the $\mathrm{S} / \mathrm{N} / \ldots / \mathrm{N} / \mathrm{S}$ junctions. These conditions permit the resolution of the system whatever the number of the metallic layers. From the general equations of the multilayer (4), we write the equations for the Green functions with an arbitrary number of metallic layers (which must not be the last layer) as:

$$
\begin{gathered}
\left(\mathrm{i} \omega-\xi_{n}\right) G_{\uparrow, \uparrow}^{n, m}-t G_{\uparrow, \uparrow}^{n-1, L}-t G_{\uparrow, \uparrow}^{n+1, m}=0 \\
\left(\mathrm{i} \omega+\xi_{n}\right) F_{\downarrow, \uparrow}^{\dagger n, m}-t F_{\downarrow, \uparrow}^{\dagger n-1, m}-t F_{\downarrow, \uparrow}^{\dagger n+1, m}=0 .
\end{gathered}
$$

To solve the system (A.1), we use the form of the Gorkov function (4) and the relations (5) and (6). We have:

$$
\begin{aligned}
\mathrm{e}^{\widetilde{q}} & =-\left(\frac{\mathrm{i} \omega+\xi_{n}}{2 t}\right)+\frac{\sqrt{\left(\mathrm{i} \omega+\xi_{n}\right)^{2}-4 t^{2}}}{2 t} \\
\mathrm{e}^{-\widetilde{q}} & =-\left(\frac{\mathrm{i} \omega+\xi_{n}}{2 t}\right)-\frac{\sqrt{\left(\mathrm{i} \omega+\xi_{n}\right)^{2}-4 t^{2}}}{2 t} \\
\mathrm{e}^{q} & =\left(\frac{\mathrm{i} \omega-\xi_{n}}{2 t}\right)+\frac{\sqrt{\left(\mathrm{i} \omega-\xi_{n}\right)^{2}-4 t^{2}}}{2 t} \\
\mathrm{e}^{-q} & =\left(\frac{\mathrm{i} \omega-\xi_{n}}{2 t}\right)-\frac{\sqrt{\left(\mathrm{i} \omega-\xi_{n}\right)^{2}-4 t^{2}}}{2 t}
\end{aligned}
$$

and we can write the explicit form of the Green functions.

\section{Gorkov functions of the $\mathrm{S} / \mathrm{N} / \ldots / \mathrm{N}$ multilayer}

We study the $\mathrm{S} / \mathrm{N} / \ldots / \mathrm{N}$ system where the $\mathrm{S}$ layer is indexed by $n=0$ and the $\mathrm{N}$ layers from $n=1$ to $n=L$ (see figure 1(a)). To determine the density of states in the last metal layer (indexed $L$ ) we have to determine the $G_{\uparrow, \uparrow}^{L, L}$ Green function. The Gorkov equations for the last metal layer read as

$$
\begin{gathered}
\left(\mathrm{i} \omega-\xi_{n}\right) G_{\uparrow, \uparrow}^{L, L}-t G_{\uparrow, \uparrow}^{L-1, L}=1 \\
\left(\mathrm{i} \omega+\xi_{n}\right) F_{\downarrow, \uparrow}^{\dagger L, L}+t F_{\downarrow, \uparrow}^{\dagger L-1, L}=0,
\end{gathered}
$$

in which we replace the Green function by the form (4) in order to write the constants $B$ and $D$ as functions of the constants $A$ and $C$. Hence the Green functions in the last metal layer $(n=L)$ read as

$$
G_{\uparrow, \uparrow}^{n, L}=A\left(\mathrm{e}^{q n}-\mathrm{e}^{2 q L} \mathrm{e}^{-q(n-2)}\right)+\frac{\mathrm{e}^{q(L-n)}}{\left(\mathrm{i} \omega-\xi_{n}-t \mathrm{e}^{q}\right)}
$$

and

$$
F_{\downarrow, \uparrow}^{\dagger n, L}=C\left(\mathrm{e}^{\widetilde{q} n}-\mathrm{e}^{2 \widetilde{q} L} \mathrm{e}^{-\widetilde{q}(n-2)}\right)
$$

Then, we have to determine the constants $A$ and $C$. Furthermore, from the function (A.4) and (A.5), we can find all the Green functions calculated for arbitrary indices $n$. Then, the Gorkov equations in the $\mathrm{S}$ layer $(n=0)$ can be written as

$$
\begin{aligned}
& \left(\mathrm{i} \omega-\xi_{n}\right) G_{\uparrow, \uparrow}^{0, L}-t G_{\uparrow, \uparrow}^{1, L}+\Delta_{0} F_{\downarrow, \uparrow}^{\dagger 0, L}=0 \\
& \left(\mathrm{i} \omega+\xi_{n}\right) F_{\downarrow, \uparrow}^{\dagger 0, L}+t F_{\downarrow, \uparrow}^{\dagger 1, L}+\Delta_{0}^{*} G_{\uparrow, \uparrow}^{0, L}=0,
\end{aligned}
$$

and in the first metallic layer $(n=1)$ as

$$
\begin{gathered}
\left(\mathrm{i} \omega-\xi_{n}\right) G_{\uparrow, \uparrow}^{1, L}-t G_{\uparrow, \uparrow}^{0, L}-t G_{\uparrow, \uparrow}^{2, L}=0 \\
\left(\mathrm{i} \omega+\xi_{n}\right) F_{\downarrow, \uparrow}^{\dagger 1, L}+t F_{\downarrow, \uparrow}^{\dagger 0, L}+t F_{\downarrow, \uparrow}^{\dagger 2, L}=0 .
\end{gathered}
$$

If we replace the Green functions in the two last systems by considering the propagation condition (A.4) and (A.5), the system becomes:

$$
\begin{gathered}
\left(\mathrm{i} \omega-\xi_{\mathrm{s}}\right) G_{\uparrow, \uparrow}^{0, L}+2 t A \mathrm{e}^{q(L+1)} \sinh (q L)+\Delta_{0} F_{\downarrow, \uparrow}^{\dagger 0, L}=\mathrm{e}^{q L} \\
\left(\mathrm{i} \omega+\xi_{\mathrm{s}}\right) F_{\downarrow, \uparrow}^{\dagger 0, L}-2 t C \mathrm{e}^{\widetilde{q}(L+1)} \sinh (\widetilde{q} L)+\Delta_{0}^{*} G_{\uparrow, \uparrow}^{0, L}=0 \\
2 t A \mathrm{e}^{q(L+1)} \sinh (q(L+1))-t G_{\uparrow, \uparrow}^{0, L}=\mathrm{e}^{q(L+1)} \\
-2 t C \mathrm{e}^{\widetilde{q}(L+1)} \sinh (\widetilde{q}(L+1))+t F_{\downarrow, \uparrow}^{\dagger 0, L}=0 .
\end{gathered}
$$

And the solutions are

$$
\begin{aligned}
& A=\frac{1}{2}\left\{\widetilde{a}_{L+1}\left(\mathrm{e}^{q(L+1)}\left(\omega^{2}+\xi_{\mathrm{s}}^{2}+\left|\Delta_{0}\right|^{2}\right)-t \mathrm{e}^{q L}\left(\mathrm{i} \omega+\xi_{\mathrm{s}}\right)\right)\right. \\
& \left.+t \widetilde{a}_{L}\left(t \mathrm{e}^{q L}+\left(\mathrm{i} \omega-\xi_{\mathrm{s}}\right) \mathrm{e}^{q(L+1)}\right)\right\}\left\{t \mathrm { e } ^ { q ( L + 1 ) } \left(t^{2} a_{L} \widetilde{a}_{L}\right.\right. \\
& -t\left[\left(\mathrm{i} \omega-\xi_{\mathrm{s}}\right) \tilde{a}_{L} a_{L+1}-\left(\mathrm{i} \omega+\xi_{\mathrm{s}}\right) a_{L} \widetilde{a}_{L+1}\right] \\
& \left.\left.+\left(\omega^{2}+\xi_{\mathrm{s}}^{2}+\left|\Delta_{0}\right|^{2}\right) a_{L+1} \widetilde{a}_{L+1}\right)\right\}^{-1} \\
& C=-\frac{1}{2}\left\{\Delta_{0}^{*} a_{1}\right\}\left\{\mathrm { e } ^ { \widetilde { q } ( L + 1 ) } \left(t^{2} a_{L} \widetilde{a}_{L}-t\left[\left(\mathrm{i} \omega-\xi_{\mathrm{s}}\right) \widetilde{a}_{L} a_{L+1}\right.\right.\right. \\
& \left.-\left(\mathrm{i} \omega+\xi_{\mathrm{s}}\right) a_{L} \widetilde{a}_{L+1}\right] \\
& \left.\left.+\left(\omega^{2}+\xi_{\mathrm{s}}^{2}+\left|\Delta_{0}\right|^{2}\right) a_{L+1} \tilde{a}_{L+1}\right)\right\}^{-1} \\
& G_{\uparrow, \uparrow}^{0, L}=\left\{\sinh (q)\left(\left(\mathrm{i} \omega+\xi_{\mathrm{s}}\right) \widetilde{a}_{L+1}-\tilde{a}_{L}\right)\right\}\left\{t^{2} a_{L} \widetilde{a}_{L}\right. \\
& -t\left[\left(\mathrm{i} \omega-\xi_{\mathrm{s}}\right) \tilde{a}_{L} a_{L+1}-\left(\mathrm{i} \omega+\xi_{\mathrm{s}}\right) a_{L} \widetilde{a}_{L+1}\right] \\
& \left.+\left(\omega^{2}+\xi_{\mathrm{s}}^{2}+\left|\Delta_{0}\right|^{2}\right) a_{L+1} \widetilde{a}_{L+1}\right\}^{-1} \\
& F_{\downarrow, \uparrow}^{\dagger 0, L}=-\left\{\Delta_{0}^{*} a_{1} \tilde{a}_{L+1}\right\}\left\{t^{2} a_{L} \widetilde{a}_{L}-t\left[\left(\mathrm{i} \omega-\xi_{\mathrm{s}}\right)\right.\right. \\
& \left.\times \tilde{a}_{L} a_{L+1}-\left(\mathrm{i} \omega+\xi_{\mathrm{s}}\right) a_{L} \tilde{a}_{L+1}\right] \\
& \left.+\left(\omega^{2}+\xi_{\mathrm{s}}^{2}+\left|\Delta_{0}\right|^{2}\right) a_{L+1} \widetilde{a}_{L+1}\right\}^{-1}
\end{aligned}
$$

where $a_{n}=\sinh (q n)$ and $\widetilde{a}_{n}=\sinh (\widetilde{q} n)$. The Green functions can be written as:

$$
\begin{aligned}
G_{\uparrow, \uparrow}^{n, L} & =\frac{1}{2}\left\{\left[\widetilde { a } _ { L + 1 } \left(\mathrm{e}^{q(L+1)}\left(\omega^{2}+\xi_{\mathrm{s}}^{2}+\left|\Delta_{0}\right|^{2}\right)\right.\right.\right. \\
& \left.-t \mathrm{e}^{q L}\left(\mathrm{i} \omega+\xi_{\mathrm{s}}\right)\right)+t \widetilde{a}_{L}\left(t \mathrm{e}^{q L}\right. \\
& \left.\left.\left.+\left(\mathrm{i} \omega-\xi_{\mathrm{s}}\right) \mathrm{e}^{q(L+1)}\right)\right]\left(\mathrm{e}^{q n}-\mathrm{e}^{2 q L} \mathrm{e}^{-q(n-2)}\right)\right\} \\
& \times\left\{t \mathrm { e } ^ { q ( L + 1 ) } \left(t^{2} a_{L} \widetilde{a}_{L}-t\left[\left(\mathrm{i} \omega-\xi_{\mathrm{s}}\right) \widetilde{a}_{L} a_{L+1}\right.\right.\right. \\
& \left.\left.\left.-\left(\mathrm{i} \omega+\xi_{\mathrm{s}}\right) a_{L} \widetilde{a}_{L+1}\right]+\left(\omega^{2}+\xi_{\mathrm{s}}^{2}+\left|\Delta_{0}\right|^{2}\right) a_{L} \widetilde{a}_{L+1}\right)\right\}^{-1} \\
& +\frac{\mathrm{e}^{q(L-n)}}{\left(\mathrm{i} \omega-\xi_{n}-t \mathrm{e}^{q}\right)}
\end{aligned}
$$




$$
\begin{aligned}
F_{\downarrow, \uparrow}^{\dagger n, L} & =-\frac{1}{2}\left\{\Delta_{0}^{*} a_{1}\left(\mathrm{e}^{\widetilde{q} n}-\mathrm{e}^{2 \widetilde{q} L} \mathrm{e}^{-\widetilde{q}(n-2)}\right)\right\}\left\{\mathrm { e } ^ { \widetilde { q } ( L + 1 ) } \left(t^{2} a_{L} \widetilde{a}_{L}\right.\right. \\
& -t\left[\left(\mathrm{i} \omega-\xi_{\mathrm{s}}\right) a_{L+1} \widetilde{a}_{L}-\left(\mathrm{i} \omega+\xi_{\mathrm{s}}\right) a_{L} \widetilde{a}_{L+1}\right] \\
& \left.\left.+\left(\omega^{2}+\xi_{\mathrm{s}}^{2}+\left|\Delta_{0}\right|^{2}\right) a_{L+1} \widetilde{a}_{L+1}\right)\right\}^{-1} .
\end{aligned}
$$

If $n=L$ then the Green function in the $L$ layer can be written as:

$$
\begin{aligned}
G_{\uparrow, \uparrow}^{L, L}= & \left\{t^{2} a_{L-1} \tilde{a}_{L}-t\left[\left(\mathrm{i} \omega-\xi_{\mathrm{s}}\right) a_{L} \tilde{a}_{L}-\left(\mathrm{i} \omega+\xi_{\mathrm{s}}\right) a_{L-1}\right.\right. \\
& \left.\left.\times \widetilde{a}_{L+1}\right]+\left(\omega^{2}+\xi_{\mathrm{s}}^{2}+\left|\Delta_{0}\right|^{2}\right) a_{L} \tilde{a}_{L+1}\right\}\left\{t \left(t^{2} a_{L} \tilde{a}_{L}\right.\right. \\
& -t\left[\left(\mathrm{i} \omega-\xi_{\mathrm{s}}\right) a_{L+1} \widetilde{a}_{L}-\left(\mathrm{i} \omega+\xi_{\mathrm{s}}\right) a_{L} \widetilde{a}_{L+1}\right] \\
& \left.\left.+\left(\omega^{2}+\xi_{\mathrm{s}}^{2}+\left|\Delta_{0}\right|^{2}\right) a_{L+1} \tilde{a}_{L+1}\right)\right\}^{-1} \\
F_{\downarrow, \uparrow}^{\dagger L, L} & =\left\{\Delta_{0}^{*} a_{1} \widetilde{a}_{1}\right\}\left\{\left(t^{2} a_{L} \widetilde{a}_{L}-t\left[\left(\mathrm{i} \omega-\xi_{\mathrm{s}}\right) a_{L+1} \tilde{a}_{L}\right.\right.\right. \\
& \left.-\left(\mathrm{i} \omega+\xi_{\mathrm{s}}\right) a_{L} \widetilde{a}_{L+1}\right] \\
& \left.\left.+\left(\omega^{2}+\xi_{\mathrm{s}}^{2}+\left|\Delta_{0}\right|^{2}\right) a_{L+1} \tilde{a}_{L+1}\right)\right\}^{-1} .
\end{aligned}
$$

The Green functions can be simplified as:

$$
\begin{aligned}
& G_{\uparrow, \uparrow}^{n, 0}=A\left(\mathrm{e}^{q n}-\mathrm{e}^{2 q L} \mathrm{e}^{-q(n-2)}\right) \\
& F_{\downarrow, \uparrow}^{\dagger n, 0}=C\left(\mathrm{e}^{\widetilde{q} n}-\mathrm{e}^{2 \widetilde{q} L} \mathrm{e}^{-\widetilde{q}(n-2)}\right)
\end{aligned}
$$

and with relation (A.4) and (A.5) this system of equation can be written as:

$$
\begin{gathered}
\left(\mathrm{i} \omega-\xi_{\mathrm{s}}\right) G_{\uparrow, \uparrow}^{0,0}+2 t A \mathrm{e}^{q(L+1)} \sinh (q L)+\Delta_{0} F_{\downarrow, \uparrow}^{\dagger 0,0}=1 \\
\left(\mathrm{i} \omega+\xi_{\mathrm{s}}\right) F_{\downarrow, \uparrow}^{\dagger \dagger, 0}-2 t C \mathrm{e}^{\widetilde{q}(L+1)} \sinh (\widetilde{q} L)+\Delta_{0}^{*} G_{\uparrow, \uparrow}^{0,0}=0 \\
2 t A \mathrm{e}^{q(L+1)} \sinh (q(L+1))-t G_{\uparrow, \uparrow}^{0,0}=0 \\
2 t C \mathrm{e}^{\widetilde{q}(L+1)} \sinh (\widetilde{q}(L+1))+t F_{\downarrow, \uparrow}^{\dagger 0,0}=0
\end{gathered}
$$

and the Green function in the superconducting layer can be written as

$$
\begin{aligned}
& F_{\downarrow, \uparrow}^{\dagger 0,0}=\left\{\Delta_{0}^{*} a_{L+1} \widetilde{a}_{L+1}\right\}\left\{\left(\omega^{2}+\xi_{\mathrm{s}}^{2}+\left|\Delta_{0}\right|^{2}\right) a_{L+1} \tilde{a}_{L+1}\right. \\
& \left.\quad-t\left[\left(\mathrm{i} \omega-\xi_{\mathrm{s}}\right) a_{L+1} \widetilde{a}_{L}-\left(\mathrm{i} \omega+\xi_{\mathrm{s}}\right) a_{L} \widetilde{a}_{L+1}\right]+t^{2} a_{L} \widetilde{a}_{L}\right\}^{-1}
\end{aligned}
$$

$$
\begin{aligned}
G_{\uparrow, \uparrow}^{0,0}= & \left\{-a_{L+1}\left[\left(\mathrm{i} \omega+\xi_{\mathrm{s}}\right) \widetilde{a}_{L+1}+t \widetilde{a}_{L}\right]\right\}\left\{\left(\omega^{2}+\xi_{\mathrm{s}}^{2}+\left|\Delta_{0}\right|^{2}\right)\right. \\
& \times a_{L+1} \widetilde{a}_{L+1}-t\left[\left(\mathrm{i} \omega-\xi_{\mathrm{s}}\right) a_{L+1} \widetilde{a}_{L}\right. \\
& \left.\left.-\left(\mathrm{i} \omega+\xi_{\mathrm{s}}\right) a_{L} \widetilde{a}_{L+1}\right]+t^{2} a_{L} \widetilde{a}_{L}\right\}^{-1} .
\end{aligned}
$$

From these Green functions, we can determine the properties of the $\mathrm{S}$ layer as a function of the total number of layers $L$.

\section{Gorkov equations in the $\mathrm{S} / \mathrm{N} / \ldots / \mathrm{N} / \mathrm{S}$ junction}

In the $\mathrm{S} / \mathrm{N} / \ldots / \mathrm{N} / \mathrm{S}$ system, the superconducting layers are labeled $n=0$ and $n=L$ whereas the metallic layers are labeled from $n=1$ to $n=L-1$. The calculation is the same as in the last section, hence, we establish the equations in the layers labeled $n=0, n=1, n=L-1$ and $n=L$ (see figure 1(b)). Hence, to determine the normal and anomalous
Green functions in the $\mathrm{S}$ layer labeled $n=L$, the system of equations, with the relation (5) and (6), becomes

$$
\begin{aligned}
& \left(\mathrm{i} \omega-\xi_{\mathrm{s}}\right) G_{\uparrow, \uparrow}^{0, L}-t\left(A \mathrm{e}^{q}+B \mathrm{e}^{-q}\right)+\Delta_{0}^{*} F_{\downarrow, \uparrow}^{\dagger 0, L}=0 \\
& \left(\mathrm{i} \omega+\xi_{\mathrm{s}}\right) F_{\downarrow, \uparrow}^{\dagger 0, L}+t\left(C \mathrm{e}^{\widetilde{q}}+D \mathrm{e}^{-\widetilde{q}}\right)+\Delta_{0} G_{\uparrow, \uparrow}^{0, L}=0 \\
& A+B-G_{\uparrow, \uparrow}^{0, L}=0 \\
& C+D-t F_{\downarrow, \uparrow}^{\dagger 0, L}=0 \\
& A \mathrm{e}^{q L}+B \mathrm{e}^{-q L}-G_{\uparrow, \uparrow}^{L, L}=0 \\
& C \mathrm{e}^{\widetilde{q} L}+D \mathrm{e}^{-\widetilde{q} L}-F_{\downarrow, \uparrow}^{\dagger L, L}=0 \\
& \left(\mathrm{i} \omega-\xi_{\mathrm{s}}\right) G_{\uparrow, \uparrow}^{L, L}-t\left(A \mathrm{e}^{(L-1) q}+B \mathrm{e}^{-(L-1) q}\right) \\
& \quad+\Delta_{L}^{*} F_{\downarrow, \uparrow}^{\dagger L, L}-1=0 \\
& \left(\mathrm{i} \omega+\xi_{\mathrm{s}}\right) F_{\downarrow, \uparrow}^{\dagger L, L}+t\left(C \mathrm{e}^{\widetilde{q}(L-1)}+D \mathrm{e}^{-\widetilde{q}(L-1)}\right) \\
& \quad+\Delta_{L} G_{\uparrow, \uparrow}^{0, L}=0 .
\end{aligned}
$$

The solution of the system (A.22) can be written as:

$$
\begin{aligned}
G_{\uparrow, \uparrow}^{L, L} & =\left\{-t^{3} S_{L-1}^{L-2}-t^{2}\left[\widetilde{\omega}^{*} S_{L}^{L-2}+2 \widetilde{\omega} S_{L-1}^{L-1}\right]\right. \\
& \left.-t\left[S_{L}^{L-1}\left(|\widetilde{\omega}|^{2}+\Omega_{0}^{2}\right)+\widetilde{\omega}^{2} S_{L-1}^{L}\right]-\widetilde{\omega} \Omega_{0}^{2} S_{L}^{L}\right\}\left\{t^{4} S_{L-2}^{L-2}\right. \\
& +2 t^{3} X_{L-2}^{L-1}+t^{2}\left[\left(2|\widetilde{\omega}|^{2}+\Omega_{0}^{2}+\Omega_{L}^{2}\right) S_{L-1}^{L-1}+2 \Delta_{0} \Delta_{L} S_{1}^{1}\right. \\
& \left.+\widetilde{\omega}^{*^{2}} S_{L-2}^{L}+\widetilde{\omega}^{2} S_{L-2}^{L}\right]+t\left(\Omega_{0}^{2}+\Omega_{L}^{2}\right) X_{L-1}^{L} \\
& \left.+S_{L}^{L} \Omega_{L}^{2} \Omega_{0}^{2}\right\}^{-1} \\
F_{\downarrow, \uparrow}^{\dagger L, L} & =\left\{t^{2} \Delta_{0}^{*} S_{1}^{1}+\Delta_{L}^{*}\left\{\Omega_{0}^{2} S_{L}^{L}+t^{2} S_{L}^{L-1}-t \widetilde{\omega}^{*} S_{L-1}^{L}\right.\right. \\
& \left.\left.-t \widetilde{\omega} S_{L-1}^{L}\right\}\right\}\left\{t^{4} S_{L-2}^{L-2}+2 t^{3} X_{L-2}^{L-1}+t^{2}\left[\left(2|\widetilde{\omega}|^{2}\right.\right.\right. \\
& \left.+\Omega_{0}^{2}+\Omega_{L}^{2}\right) S_{L-1}^{L-1}+2 \Delta_{0} \Delta_{L} S_{1}^{1}+\widetilde{\omega}^{*^{2}} S_{L-2}^{L} \\
& \left.\left.+\widetilde{\omega}^{2} S_{L-2}^{L}\right]+t\left(\Omega_{0}^{2}+\Omega_{L}^{2}\right) X_{L-1}^{L}+S_{L}^{L} \Omega_{L}^{2} \Omega_{0}^{2}\right\}^{-1} \\
G_{\uparrow, \uparrow}^{0, L} & =\left\{-t^{3} S_{1}^{L-2}-2 t^{2} \widetilde{\omega} S_{1}^{L-1}-t\left[S_{1}^{L} \widetilde{\omega}^{2}+\Delta_{0}^{*} \Delta_{2}^{*} S_{L}^{1}\right]\right\} \\
& \times\left\{t^{4} S_{L-2}^{L-2}+2 t^{3} X_{L-2}^{L-1}+t^{2}\left[\left(2|\widetilde{\omega}|^{2}+\Omega_{0}^{2}+\Omega_{L}^{2}\right) S_{L-1}^{L-1}\right.\right. \\
& \left.+2 \Delta_{0} \Delta_{L} S_{1}^{1}+\widetilde{\omega}^{*} S_{L-2}^{L}+\widetilde{\omega}^{2} S_{L-2}^{L}\right]+t\left(\Omega_{0}^{2}\right. \\
& \left.\left.+\Omega_{L}^{2}\right) X_{L-1}^{L}+S_{L}^{L} \Omega_{L}^{2} \Omega_{0}^{2}\right\}^{-1}
\end{aligned}
$$

$F_{\downarrow, \uparrow}^{\dagger 0, L}=\left\{t^{2}\left[\Delta_{0}^{*} S_{1}^{L-1}+\Delta_{2}^{*} S_{L-1}^{1}\right]+t \Delta_{0}^{*} S_{1}^{L} \widetilde{\omega}-t \Delta_{2}^{*} \widetilde{\omega}^{*} S_{L}^{1}\right\}$

$$
\begin{aligned}
& \times\left\{t^{4} S_{L-2}^{L-2}+2 t^{3} X_{L-2}^{L-1}+t^{2}\left[\left(2|\widetilde{\omega}|^{2}+\Omega_{0}^{2}+\Omega_{L}^{2}\right) S_{L-1}^{L-1}\right.\right. \\
& \left.+2 \Delta_{0} \Delta_{L} S_{1}^{1}+\widetilde{\omega}^{*^{2}} S_{L-2}^{L}+\widetilde{\omega}^{2} S_{L-2}^{L}\right] \\
& \left.+t\left(\Omega_{0}^{2}+\Omega_{L}^{2}\right) X_{L-1}^{L}+S_{L}^{L} \Omega_{L}^{2} \Omega_{0}^{2}\right\}^{-1}
\end{aligned}
$$

$A=\left\{-t^{3} \mathrm{e}^{-q} \widetilde{a}_{L-2}+t^{2}\left[2 \widetilde{\omega} \mathrm{e}^{q}-\widetilde{\omega}^{*} \mathrm{e}^{-\widetilde{q}}\right] \widetilde{a}_{L-1}\right.$

$$
\begin{aligned}
& +t\left[-\Delta_{2}^{*} \Delta_{0}^{*} \mathrm{e}^{-q L} \widetilde{a}_{1}+\mathrm{e}^{-q} \widetilde{a}_{L} \widetilde{\omega}^{2}+\left(\xi_{\mathrm{s}}^{2}-\omega^{2}\right) \mathrm{e}^{-\widetilde{q}(L-1)}\right. \\
& \left.\left.+|\widetilde{\omega}|^{2} \mathrm{e}^{\widetilde{q}(L-1)} \widetilde{a}_{L-1}-\Omega_{0}^{2} \widetilde{a}_{L-1}\right]+\widetilde{a}_{L} \widetilde{\omega} \Omega_{0}^{2}\right\}\left\{2 \left[t^{4} S_{L-2}^{L-2}\right.\right.
\end{aligned}
$$




$$
\begin{aligned}
& +2 t^{3} X_{L-2}^{L-1}+t^{2}\left[\left(2|\widetilde{\omega}|^{2}+\Omega_{0}^{2}+\Omega_{L}^{2}\right) S_{L-1}^{L-1}+2 \Delta_{0} \Delta_{L} S_{1}^{1}\right. \\
& \left.+\widetilde{\omega}^{*^{2}} S_{L-2}^{L}+\widetilde{\omega}^{2} S_{L-2}^{L}\right]+t\left(\Omega_{0}^{2}+\Omega_{L}^{2}\right) X_{L-1}^{L} \\
& \left.\left.+S_{L}^{L} \Omega_{L}^{2} \Omega_{0}^{2}\right]\right\}^{-1} \\
B= & \left\{-t^{3} \mathrm{e}^{-q} \widetilde{a}_{L-2}-t^{2}\left[2 \widetilde{\omega} \mathrm{e}^{-q}-\widetilde{\omega}^{*} \mathrm{e}^{\widetilde{q}}\right] \widetilde{a}_{L-1}\right. \\
& +t\left[-\Delta_{2}^{*} \Delta_{0}^{*} \mathrm{e}^{q L} \widetilde{a}_{1}+\mathrm{e}^{q} \widetilde{a}_{L} \widetilde{\omega}^{2}+\left(\xi_{\mathrm{S}}^{2}-\omega^{2}\right) \mathrm{e}^{\widetilde{q}(L-1)}\right. \\
& \left.\left.+|\widetilde{\omega}|^{2} \mathrm{e}^{-\widetilde{q}(L-1)} \widetilde{a}_{L-1}-\Omega_{0}^{2} \widetilde{a}_{L-1}\right]-\widetilde{a}_{L} \widetilde{\omega} \Omega_{0}^{2}\right\}\left\{2 \left[t^{4} S_{L-2}^{L-2}\right.\right. \\
& +2 t^{3} X_{L-2}^{L-1}+t^{2}\left[\left(2|\widetilde{\omega}|^{2}+\Omega_{0}^{2}+\Omega_{L}^{2}\right) S_{L-1}^{L-1}+2 \Delta_{0} \Delta_{L} S_{1}^{1}\right. \\
& \left.+\widetilde{\omega}^{*^{2}} S_{L-2}^{L}+\widetilde{\omega}^{2} S_{L-2}^{L}\right]+t\left(\Omega_{0}^{2}+\Omega_{L}^{2}\right) X_{L-1}^{L} \\
& \left.\left.+S_{L}^{L} \Omega_{L}^{2} \Omega_{0}^{2}\right]\right\}^{-1} \\
C= & \left\{-t^{2}\left(\Delta_{2}^{*} \mathrm{e}^{-\widetilde{q}} a_{L-1}+\Delta_{0}^{*} \mathrm{e}^{-\widetilde{q}(L-1)} a_{1}\right)+t\left[-\Delta_{0}^{*} \widetilde{\omega} \mathrm{e}^{-L \widetilde{q}} a_{1}\right.\right. \\
& \left.\left.+\Delta_{2}^{*} \widetilde{\omega}^{*} \mathrm{e}^{-\widetilde{q}} a_{L}+\widetilde{\omega} \Delta_{2}^{*} a_{L-1}\right]-\Delta_{2}^{*} a_{L} \Omega_{0}^{2}\right\}\left\{2 \left[t^{4} S_{L-2}^{L-2}\right.\right. \\
& +2 t^{3} X_{L-2}^{L-1}+t^{2}\left[\left(2|\widetilde{\omega}|^{2}+\Omega_{0}^{2}+\Omega_{L}^{2}\right) S_{L-1}^{L-1}+2 \Delta_{0} \Delta_{L} S_{1}^{1}\right. \\
& \left.+\widetilde{\omega}^{*} S_{L-2}^{L}+\widetilde{\omega}^{2} S_{L-2}^{L}\right]+t\left(\Omega_{0}^{2}+\Omega_{L}^{2}\right) X_{L-1}^{L} \\
& \left.\left.+S_{L}^{L} \Omega_{L}^{2} \Omega_{0}^{2}\right]\right\}^{-1} \\
D= & \left\{-t^{2}\left(\Delta_{2}^{*} \mathrm{e}^{-\widetilde{q}} a_{L-1}+\Delta_{0}^{*} \mathrm{e}^{-\widetilde{q}(L-1)} a_{1}\right)+t\left[\Delta_{0}^{*} \widetilde{\omega}^{*} \mathrm{e}^{-L \widetilde{q}} a_{1}\right.\right. \\
& \left.\left.-\Delta_{2}^{*} \widetilde{\omega}^{*} \mathrm{e}^{\widetilde{q}} a_{L}-\widetilde{\omega} \Delta_{2}^{*} a_{L-1}\right]+\Delta_{2}^{*} a_{L} \Omega_{0}^{2}\right\}\left\{2 \left[t^{4} S_{L-2}^{L-2}\right.\right. \\
& +2 t^{3} X_{L-2}^{L-1}+t^{2}\left[\left(2|\widetilde{\omega}|^{2}+\Omega_{0}^{2}+\Omega_{L}^{2}\right) S_{L-1}^{L-1}\right. \\
& \left.+2 \Delta_{0} \Delta_{L} S_{1}^{1}+\widetilde{\omega}^{*} S_{L-2}^{L}+\widetilde{\omega}^{2} S_{L-2}^{L}\right] \\
& \left.\left.+t\left(\Omega_{0}^{2}+\Omega_{L}^{2}\right) X_{L-1}^{L}+S_{L}^{L} \Omega_{L}^{2} \Omega_{0}^{2}\right]\right\}^{-1} \\
&
\end{aligned}
$$

where $a_{n}=\sinh (q n)$ and $\widetilde{a}_{n}=\sinh (\widetilde{q} n), S_{\mathrm{N}}^{\mathrm{M}}=\widetilde{a}_{\mathrm{M}} a_{\mathrm{N}}, \widetilde{\omega}=$ $\mathrm{i} \omega+\xi_{\mathrm{s}}, \widetilde{\omega}^{*}=-\mathrm{i} \omega+\xi_{\mathrm{s}}=-\left(\mathrm{i} \omega-\xi_{\mathrm{s}}\right), \Omega_{0}^{2}=|\widetilde{\omega}|^{2}+\Delta_{0}^{2}$, $\Omega_{L}^{2}=|\widetilde{\omega}|^{2}+\Delta_{L}^{2}$ et $X_{\mathrm{M}}^{N}=\widetilde{\omega} S_{\mathrm{M}}^{N}+\widetilde{\omega}^{*} S_{\mathrm{N}}^{\mathrm{M}}$. The normal and anomalous Green functions in the first $\mathrm{S}$ layer can be written as

$$
\begin{aligned}
G_{\uparrow, \uparrow}^{0,0} & =\left\{-t^{3} S_{L-1}^{L-2}-t^{2}\left[\widetilde{\omega}^{*} S_{L}^{L-2}+2 \widetilde{\omega} S_{L-1}^{L-1}\right]-t\left[S _ { L } ^ { L - 1 } \left(|\widetilde{\omega}|^{2}\right.\right.\right. \\
& \left.\left.\left.+\Omega_{L}^{2}\right)+\widetilde{\omega}^{2} S_{L-1}^{L}\right]-\widetilde{\omega} \Omega_{L}^{2} S_{L}^{L}\right\}\left\{t^{4} S_{L-2}^{L-2}+2 t^{3} X_{L-2}^{L-1}\right. \\
& +t^{2}\left[\left(2|\widetilde{\omega}|^{2}+\Omega_{0}^{2}+\Omega_{L}^{2}\right) S_{L-1}^{L-1}+2 \Delta_{0} \Delta_{L} S_{1}^{1}+\widetilde{\omega}^{*^{2}} S_{L-2}^{L}\right. \\
& \left.\left.+\widetilde{\omega}^{2} S_{L-2}^{L}\right]+t\left(\Omega_{0}^{2}+\Omega_{L}^{2}\right) X_{L-1}^{L}+S_{L}^{L} \Omega_{L}^{2} \Omega_{0}^{2}\right\}^{-1} \\
F_{\downarrow, \uparrow}^{\dagger 0,0} & =\left\{t^{2} \Delta_{L}^{*} S_{1}^{1}+\Delta_{0}^{*}\left\{\Omega_{L}^{2} S_{L}^{L}+t^{2} S_{L}^{L-1}-t \widetilde{\omega}^{*} S_{L-1}^{L}\right.\right. \\
& \left.\left.-t \widetilde{\omega} S_{L-1}^{L}\right\}\right\}\left\{t^{4} S_{L-2}^{L-2}+2 t^{3} X_{L-2}^{L-1}+t^{2}\left[\left(2|\widetilde{\omega}|^{2}+\Omega_{0}^{2}\right.\right.\right. \\
& \left.\left.+\Omega_{L}^{2}\right) S_{L-1}^{L-1}+2 \Delta_{0} \Delta_{L} S_{1}^{1}+\widetilde{\omega}^{*^{2}} S_{L-2}^{L}+\widetilde{\omega}^{2} S_{L-2}^{L}\right] \\
& \left.+t\left(\Omega_{0}^{2}+\Omega_{L}^{2}\right) X_{L-1}^{L}+S_{L}^{L} \Omega_{L}^{2} \Omega_{0}^{2}\right\}^{-1}
\end{aligned}
$$

where $a_{n}=\sinh (q n)$ and $\widetilde{a}_{n}=\sinh (\widetilde{q} n)$.

\section{References}

[1] Bednorz J and Mueller K 1986 Z. Phys. B 64 189-93

[2] Cyrot M and Pavuna D 1995 Introduction to Superconductivity and High $T_{\mathrm{c}}$ Materials (Singapore: World Scientific)

[3] Martin S, Fiory A T, Fleming R M, Schneemeyer L F and Waszczak J V 1988 Phys. Rev. Lett. 602194

[4] Cooper R, Forro L and Keszeit B 1990 Nature 343444

[5] Hidaka Y, Enomoto Y, Suzuki M, Oda M and Murakami T 1987 Japan. J. Appl. Phys. 26 L377

[6] Nachtrab T, Bernhard Ch, Lin Ch, Koelle D and Kleiner R 2006 C. R. Phys. 768

[7] Ive Y, Tamegai T, Sakakibara T, Goto T, Miura N, Takeya H and Takei H 1988 Physica C: Superconductivity 153-155 26

[8] Welp U, Kwok W K, Crabtree G W, Vandervoort K G and Liu J Z 1989 Phys. Rev. Lett. 621908

[9] Oda M, Hidaka Y, Suzuki M and Murakami T 1988 Phys. Rev. B 38252

[10] Tuominen M, Goldman A M, Chang Y Z and Jiang P Z 1990 Phys. Rev. B 42412

[11] Triscone J M, Karkut M G, Antognazza L, Brunner O and Fischer O 1989 Phys. Rev. Lett. 631016

[12] Triscone J M, Fischer O, Brunner O, Antognazza L, Kent A D and Karkut M G 1990 Phys. Rev. Lett. 64804

[13] Buzdin A and Bulaevskii L N 1984 Sov. Phys.-Usp. 27830 Buzdin A and Bulaevskii L N 1984 Usp. Fiz. Nauk 144415

[14] Singleton J 2000 Rep. Prog. Phys. 631111

[15] Buzdin A I 2005 Rev. Mod. Phys. 77 935-76

[16] Ginzburg V L 1952 Zh. Eksp. Teor. Fiz. 23236

[17] Lawrence W E and Doniach S 1971 Proc. 12th Int. Conf. on Low Temperature Physics, Kyoto (Keigaku, Tokyo, 1970) ed E Kanda, p 361

[18] Tinkham M 1996 Introduction to Superconductivity (New York: Dover)

[19] Meissner H 1960 Phys. Rev. 117672

[20] Smith P H, Shapiro S, Miles J L and Nicol J 1961 Phys. Rev. Lett. 6686

[21] de Gennes P G 1964 Rev. Mod. Phys. 36 225-37

[22] Andreev A 1964 Sov. Phys._JETP 19 1228-31

[23] Saint-James D 1964 J. Phys. France 25 899-905

[24] Tagirov L R, Garifullin I A, Garif' yanov N N, Khlebnikov S Ya, Tikhonov D A, Westerholt K and Zabe H 2002 J. Magn. Magn. Mater. 240577

[25] Lazar L, Westerholt K, Zabel H, Tagirov L R, Goryunov Yu V, Garif'yanov N N and Garifullin I A 2000 Phys. Rev. B 613711

[26] Ryazanov V V, Oboznov V A, Rusanov A Yu, Veretennikov A V, Golubov A A and Aarts J 2001 Phys. Rev. Lett. 86 2427-30

[27] Golubov A A, Kupriyanov M Yu and Il'Chev E 2004 Rev. Mod. Phys. 76 411-69

[28] Bergeret F S, Volkov A F and Efetov K B 2005 Rev. Mod. Phys. 771321

[29] Fominov Ya V, Chtchelkatchev N M and Golubov A A 2001 JETP Lett. 7496

[30] Josephson B D 1962 Phys. Lett. 1251

[31] Giaever I and Megerle K 1961 Phys. Rev. 1221101

[32] Montiel X and Buzdin A I 2011 Phys. Rev. B 84054518

[33] Tollis S, Cayssol J and Buzdin A I 2006 Phys. Rev. B 73174519

[34] Yuasa R, Nemoto M, Fujiwara S, Furukawa H, Mukaida H, Tokunaga S and Nakao M 1991 Physica C: Superconductivity 185-189 2587-8

[35] Tarutani Y, Fukazawa T, Kabasawa U, Tsukamoto A, Hiratani M and Takagi K 1991 Appl. Phys. Lett. 582707

[36] Barner J B, Rogers C T, Inam A, Ramesh R and Bersey S 1991 Appl. Phys. Lett. 59742 
[37] Kasai M, Kanke Y, Ohno T and Kozono Y 1992 J. Appl. Phys. 725344

[38] Meltzow A C, Hu S, HollKott J, Auge J, Spangenberg B, Kurz H, Zakharov N D and Hesse D 1997 IEEE Trans. Appl. Supercond. 72852

[39] Decca R S, Drew H D, Osquiguil E, Maiorov B and Guimpel J 2000 Phys. Rev. Lett. 853708

[40] Sefrioui Z, Arias D, Peña V, Villegas J E, Varela M, Prieto P, León C, Martinez J L and Santamaria J 2003 Phys. Rev. B 67214511

[41] Delin K A and Kleinsasser A W 1996 Supercond. Sci. Technol. $9227-69$

[42] Kresin Y, Ovchinikov Yu and Wolf S 2003 Appl. Phys. Lett. 83722

[43] Bozovic I, Logvenov G, Verhoeve M A J, Caputo P, Goldobin E and Beasley M R 2004 Phys. Rev. Lett. 93157002

[44] Bergeret F S, Volkov A F and Efetov K B 2001 Phys. Rev. Lett. 86 4096-9

[45] Buzdin A I, Damjanović V P and Simonov A Yu 1992 Phys. Rev. B 45 7499-502

[46] Liu S H and Klemm R A 1994 Phys. Rev. Lett. 73 1019-22

[47] Belzig W, Bruder C and Schön G 1996 Phys. Rev. B 549443
[48] Radović Z, Ledvij M, Dobrosavljević-Grujić L, Buzdin A I and Clem J R 1991 Phys. Rev. B 44759

[49] Gupta A K, Crétinon L, Moussy N, Pannetier B and Courtois H 2004 Phys. Rev. B 69104514

[50] Montiel X, Gusakova D, Daumens M and Buzdin A I 2009 Europhys. Lett. 8967002

[51] Eilenberger G 1968 Z. Phys. 214195

[52] Usadel K D 1970 Phys. Rev. Lett. 25507

[53] Andreev A V, Buzdin A I and Osgood R M III 1991 Phys. Rev. B 4310124

[54] Bulaevskii L N, Buzdin A I, Panjukov S V and Kulic M L 1983 Phys. Rev. B 281370

[55] Bulaevskii L N, Osgood R M III and Zyskin M V $1990 \mathrm{~J}$. Phys.: Condens. Matter 2 7867-72

[56] Bergeret F S, Levy Yeyati A and Martín-Rodero A 2005 Phys. Rev. B 72064524

[57] Abrikosov A A, Gorkov L P and Dzyaloshinski I E 1963 Methods of Quantum Field Theory in Statistical Physics (New Jersey: Prentice-Hall)

[58] Hsiang T Y and Finnemore D K 1980 Phys. Rev. B 22154

[59] Prokic V, Buzdin A I and Dobrosavljevic-Grujic L 1999 Phys. Rev. B 59587 